\title{
Modeling and Experiments of Binary Electrolytes in the Presence of Diffusion, Migration, and Electro-Osmotic Flow
}

\author{
J.W. Haverkort $\oplus^{*}$ \\ Delft University of Technology, Process \& Energy Department, Leeghwaterstraat 39, 2628 CB Delft, The \\ Netherlands
}

(Received 25 March 2020; revised 10 August 2020; accepted 3 September 2020; published 26 October 2020)

\begin{abstract}
Combined diffusion, migration, and advection of ions in a binary electrolyte plays a role in various applications, including water electrolysis, electrodeposition, deionization, and electrophoresis. Here we analyze a dilute binary electrolyte with arbitrary ion valencies in a porous or nonporous medium using the one-dimensional Nernst-Planck equations. We examine how advection influences the limiting current, diffusion potential, and overall potential, deriving broadly useful analytical expressions. We provide experimental results for the electro-osmotic flow through a submicroporous separator in an alkaline water electrolysis setup. The time evolution of the potential is followed from the initial timescale of double-layer charging, followed by the diffusional timescale, to the time at which a limiting current is reached. For the longer timescales, a quasisteady model is shown to predict the time evolution of the advection-modified potential drop reasonably well. Additional interesting features arising due to electro-osmotic drag and unsteady diffusion are observed and explained.
\end{abstract}

DOI: 10.1103/PhysRevApplied.14.044047

\section{INTRODUCTION}

Transport of strong binary electrolytes or single salts is ubiquitous in both biological systems as well as industrial applications. A description in terms of two ion species is often a useful approximation to describe transport in biological cell membranes [1-3], electro-osmotic pumps [4], or deionization techniques including electrodialysis $[5,6]$, and pressure-driven processes like reverse osmosis, ultrafiltration, and nanofiltration [7,8]. Also, in various electrochemical systems binary electrolytes are used; for example, in water electrolysis $[9,10]$, electroplating or electrodeposition [11,12], and redox flow batteries [13]. In these applications one of the ions reacts so that to reach a desired reaction rate or current density requires sufficient mass transfer. Except perhaps in desalination, where they are the goal, concentration differences are typically undesirable. Adverse effects include increased ohmic resistance and activation overpotentials, collectively referred

\footnotetext{
*j.w.haverkort@tudelft.nl; https://jwhaverkort.weblog.tude lft.nl/

Published by the American Physical Society under the terms of the Creative Commons Attribution 4.0 International license. Further distribution of this work must maintain attribution to the author(s) and the published article's title, journal citation, and DOI.
}

to as ion concentration polarization. Flows can enhance or decrease concentration gradients and thus influence polarization and limiting currents [6,14-16]. Flows can arise due to natural convection [6], active pumping [4,7], electro-osmosis or ion drag [16,17], or deposition layer growth [11,12].

Many studies in this area consider charged, nanoporous, ion-selective membranes in which diffusio-osmosis [18] and surface-conduction $[14,19]$ play a role and electroosmotic or electroconvective instabilities can arise, allowing over-limiting currents beyond the limiting current [19-23]. Here we focus on porous media with only a very weak surface charge, sometimes referred to as "leaky membranes" [24,25] that have a part in various applications [26,27]. Here we only consider "ideally leaky" [25], very weakly charged, or nearly neutral media, as quantified in Eq. (1).

We start in Sec. II C by deriving a convenient analytical expression for the potential drop over a layer in the presence of diffusion, migration, and advection. In Sec. II D we use this potential drop expression in an equation that describes the slow time dynamics of the electrolyte concentration, resulting from both flow and a reaction. Next, in Sec. III, we show the results of experiments in the context of alkaline water electrolysis that at least partially validate the discussed models. Finally, in Sec. IV we discuss in which aspects the experiments deviate from the models, which exposes several interesting additional physical phenomena. 


\section{THEORY}

\section{A. Assumptions}

\section{Electroneutrality}

For a binary electrolyte with cation and anion valencies $z_{+}$and $z_{-}$and concentrations $c_{+}$and $c_{-}$, respectively, the mobile charge density reads $F\left(z_{+} c_{+}+z_{-} c_{-}\right)$. In most works studying electro-osmotic flow, additionally a fixed surface charge density is considered. Here we assume that the dimensionless fixed-charge density is small so that

$$
\tilde{\rho}_{s} \equiv \frac{\rho_{s}}{2 F z_{+} c_{+}} \ll 1
$$

The parameter $\tilde{\rho}_{s}$ is used, for example, in Refs. [25,28] where good membranes are assumed to have $\tilde{\rho}_{s} \gg 1$, while leaky membranes have either $\tilde{\rho}_{s}$ of order unity or $\tilde{\rho}_{s} \ll 1$. When Eq. (1) holds, we can write charge neutrality as

$$
z_{+} c_{+}+z_{-} c_{-}=0 .
$$

The fixed charge density can be crudely estimated as $\rho_{s} \sim$ $a C \zeta$ with $a C$ the volumetric capacity of the material and $\zeta$ the zeta potential. The separators used in the experiments of Sec. III have a volumetric surface area $a \sim 2 \times$ $10^{7} \mathrm{~m}^{2} / \mathrm{m}^{3}$ [29], a typical areal capacity of $C \sim 1 \mathrm{~F} / \mathrm{m}^{2}$ [30] and $\zeta \sim 50 \mathrm{mV}$ [31]. This gives $\rho_{s} / F \sim 10 \mathrm{mM}$. Since in practical applications concentrations are usually in the molar range, for these types of porous materials, a finite space charge can be safely neglected. Consequently, we can also neglect surface conduction relative to bulk conduction. However, as also remarked in Ref. [25], near limiting current conditions, the concentration strongly decreases so that a fixed charge can still significantly impact the dynamics, even when in equilibrium $\tilde{\rho}_{s} \ll 1$ holds.

\section{Pore size}

We use macrohomogeneous equations obtained from averaging over many pores. Therefore, the typical pore size should be much smaller than the porous layer thickness, often of the order of a millimeter. On the other hand, we neglect overlapping double layer effects and the contribution of surface conduction. This requires pore sizes much larger than the Debye length, typically of the order of a nanometer in the molar concentration range. Alkaline water electrolyser separators have a pore size of the order of $10^{-7} \mathrm{~m}$, comfortably satisfying both these conditions. In combination with a weak space charge, electro-osmotic or electroconvective instabilities can arise near a charge selective surface [20-23] allowing over-limiting currents beyond the limiting current. The numerical results of Ref. [19], for example, hint that instabilities may also play a role in the alkaline water separators used in Sec. III, although they will only be relevant when limiting conditions are actually reached.

\section{Governing equations}

The Nernst-Planck equations for a dilute species $i$ with molar concentration $c_{i}$ and molar flux $\mathbf{N}_{i}$ read [32]

$$
\frac{\partial \epsilon c_{i}}{\partial t}=-\nabla \cdot \mathbf{N} \quad \text { with } \mathbf{N}=\mathbf{u} c_{i}-D_{i}\left(\nabla c_{i}+\frac{z_{i} F}{R T} c_{i} \nabla \phi\right) .
$$

Here, $\mathbf{u}$ is the superficial velocity, the volumetric flow rate per total unit of geometrical area; or, equivalently, the average interstitial velocity multiplied by the porosity $\epsilon$. The absolute temperature is denoted with $T$, and $F / R \approx 11605$ $\mathrm{K} / \mathrm{V}$ is the ratio of Faraday's constant and the gas constant —or the ratio $e / k_{B}$ of the elementary charge and Boltzmann's constant. The electrostatic potential in the electrolyte is denoted with $\phi$. Note that we do not use Poisson's equation for $\phi$, since this is effectively replaced by Eq. (2) expressing electroneutrality [32].

The superficial velocity $\mathbf{u}$ is assumed to be driven by an electric field $-\nabla \phi$ and a pressure gradient $\nabla p$ according to

$$
\mathbf{u}=-\mathcal{M} \nabla \phi-\frac{k}{\mu} \nabla p
$$

The second term on the right-hand side represents Darcy's equation, with $k$ the medium permeability. In the dilute limit, the electro-osmotic mobility $\mathcal{M}$, neglecting surface viscosity, is given by the Helmholtz-Smoluchowski relation $[4,17,19,33,34]$ as

$$
\mathcal{M}=-\epsilon \frac{\varepsilon_{r} \varepsilon_{0} \zeta}{\mu}
$$

Here $\varepsilon_{0} \varepsilon_{r}$ is the electrical permittivity of the electrolyte, with $\varepsilon_{r}$ the relative permittivity compared to that of the vacuum $\varepsilon_{0} \approx 8.854 \mathrm{~F} / \mathrm{m}$. The porosity $\epsilon$ enters because we defined the mobility with respect to the superficial velocity, $\mu$ is the liquid bulk viscosity, and $\zeta$ the zeta potential [17, 19]. Here we use $\zeta$ as merely an empirical parameter that can be considered to be defined by Eq. (5).

For an incompressible fluid, the superficial velocity $u$ in the $x$ direction of Fig. 1 will be constant. Integrating the $x$ component of Eq. (4) over the thickness $L$ gives

$$
u=-\mathcal{M} \frac{\Delta \phi}{L}-\frac{k}{\mu} \frac{\Delta p}{L},
$$

where $-\Delta \phi / L$ and $\Delta p / L$ are the average electric field strength and pressure gradient in the $x$ direction, respectively. When the net pressure difference $\Delta p \equiv p(L)-$ $p(0)$ vanishes, pressure seemingly plays no role. Inside the medium, however, the electric field will not be constant. Internal pressure gradients will ensure that Eq. (4) holds. 


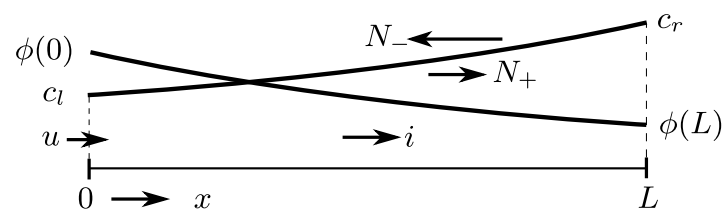

FIG. 1. A schematic of the electrolyte concentration $c$ and potential $\phi$ in the case of a positive superficial velocity $u$ and current density $i$ in the $x$ direction, resulting from ion fluxes $N_{+}$ and $N_{-}$.

\section{Material properties}

In the infinitely dilute approximation, the molecular diffusivity $D_{i \infty}$ of species $i$ in an unconfined liquid is taken to be a constant. We note that the dilute approximation can also often be used to a reasonable degree of accuracy for finite concentrations, sometimes up to several molar. Inside a porous medium with porosity $\epsilon$ and tortuosity $\tau$, the diffusivity is effectively modified to

$$
D_{i}=D_{i \infty} \epsilon / \tau,
$$

which is used in Eq. (3). Bruggeman's equation, for a packed bed of spherical particles, provides a correlation $\tau \approx 1 / \sqrt{\epsilon}$ so that $D_{i} \approx D_{i \infty} \epsilon^{1.5}$. With constant porous medium properties, in the dilute approximation, $D_{i}$ can therefore be taken to be constant. In principle, the equations of Sec. IIC can also be applied to a single thin nonporous channel using $\epsilon=\tau=1$, as long as the foregoing assumptions on pore size hold for the channel diameter. Note that the effect of Taylor dispersion on $D_{i}$ can safely be neglected, unless the Péclet number based on the pore size as a length scale becomes much larger than unity.

\section{B. Model equations}

We consider the dissociation $\mathrm{AC} \rightarrow v_{-} A^{z_{-}}+v_{+} C^{z_{+}}$of a salt $\mathrm{AC}$ into a strong electrolyte solution of anions $A^{z_{-}}$ and cations $C^{z_{+}}$, where the charge numbers or valencies $z_{+}>0$ and $z_{-}<0$. Conservation of charge requires

$$
v_{+} z_{+}+v_{-} z_{-}=0
$$

Under the assumption of electroneutrality, Eq. (2), we can define a single electrolyte concentration $\left(\mathrm{mol} / \mathrm{m}^{3}\right)$

$$
c \equiv \frac{c_{+}}{v_{+}}=\frac{c_{-}}{v_{-}} .
$$

For a binary electrolyte, the species index assumes the values $i=+$ or - . The $x$ component $N_{i}$ of the flux in Eq. (3) reads

$$
N_{i}=u c_{i}-D_{i}\left(c_{i}^{\prime}+z_{i} c_{i} \frac{F \phi^{\prime}}{R T}\right)
$$

Here a prime denotes a derivative with respect to the $x$ coordinate. We introduce the zero-concentration-gradient ion transport numbers or transference numbers [32]

$$
t_{i} \equiv \frac{\left|z_{i}\right| D_{i}}{z_{+} D_{+}-z_{-} D_{-}}
$$

satisfying $t_{-}+t_{+}=0$. In steady state, the $x$ component of Eq. (3) gives $N_{i}^{\prime}=0$. For constant $t_{i}$, the linear combination $N \equiv t_{-} N_{+} / \nu_{+}+t_{+} N_{-} / \nu_{-}$therefore also satisfies $N^{\prime}=0$, with the salt flux $N$ given by

$$
N=u c-D_{a} c^{\prime}
$$

Here the salt diffusivity, or ambipolar diffusivity, is given by

$$
D_{a} \equiv t_{-} D_{+}+t_{+} D_{-}=\frac{\left(z_{+}-z_{-}\right) D_{+} D_{-}}{z_{+} D_{+}-z_{-} D_{-}}
$$

where in the second equality we used Eq. (11). The $x$ component of the current density is given by

$$
i \equiv F\left(z_{+} N_{+}+z_{-} N_{-}\right)
$$

From the definitions of $N$ and $i$ we can write, using Eq. (8),

$$
N_{i}=N v_{i}+\frac{t_{i} i}{F z_{i}}
$$

Inserting Eq. (10) into Eq. (14), using Eq. (8), gives

$$
i=-\kappa\left(\phi^{\prime}+\chi \frac{c^{\prime}}{c}\right)
$$

The diffusion current, proportional to the parameter

$$
\chi \equiv \frac{R T}{F} \frac{D_{+}-D_{-}}{z_{+} D_{+}-z_{-} D_{-}},
$$

vanishes in the case of a symmetric electrolyte with $D_{+}=$ $D_{-}$, leaving Ohm's law $i=-\kappa \phi^{\prime}$. The electrolyte conductivity is given by

$$
\kappa=z_{+} v_{+} \frac{F^{2}}{R T}\left(z_{+} D_{+}-z_{-} D_{-}\right) c .
$$

Note that with Eqs. (8) and (9) we can rewrite Eq. (18) using $z_{+} v_{+} c=z_{+} c_{+}=-z_{-} c_{-}=-v_{-} z_{-} c$.

\section{Steady-state analytical solutions}

Solving $N^{\prime}=0$ with $c(0)=c_{l}$ on the left and $c(L)=c_{r}$ on the right, as indicated in Fig. 1, gives the well-known 
solution to the advection-diffusion equation

$$
\frac{c(x)-c_{l}}{c_{r}-c_{l}}=\frac{e^{\mathrm{Pe}(x / L)}-1}{e^{\mathrm{Pe}}-1} \stackrel{|\mathrm{Pe}| \ll 1}{\longrightarrow} \frac{x}{L},
$$

where the Péclet number is defined as

$$
\mathrm{Pe} \equiv \frac{u L}{D_{a}}
$$

We see from Eq. (19) or Fig. 2 that, for large $|\mathrm{Pe}|$, a steep exponential concentration profile arises, with most variation near $x=L$ for flow to the right, or near $x=0$ for flow to the left. In terms of the salt flux $N$ of Eq. (12) we can write $c(x)=N / u+\left(c_{r}-c_{l}\right)\left[e^{\mathrm{Pe}(x / L)} /\left(e^{\mathrm{Pe}}-1\right)\right]$ so that

$$
N=u \frac{c_{l} e^{\mathrm{Pe}}-c_{r}}{e^{\mathrm{Pe}}-1} \approx \begin{cases}u c_{l}, & \mathrm{Pe} \gg 1, \\ D_{a} \frac{c_{l}-c_{r}}{L}, & |\mathrm{Pe}| \ll 1, \\ u c_{r}, & \mathrm{Pe} \ll-1,\end{cases}
$$

which is constant over the domain. Integrating Eq. (16) gives, using Eq. (19),

$$
\phi(x)-\phi(0)=-\chi \ln \left(\frac{c(x)}{c_{l}}\right)-\frac{i L}{\kappa_{\mathrm{c}}}\left(\frac{x}{L}-\frac{\ln \left[c(x) / c_{l}\right]}{\mathrm{Pe}}\right) .
$$

Here the first term represents the diffusion potential, whose effect can be seen in Fig. 3. The second part is the ohmic potential drop, which consists of a linear as well

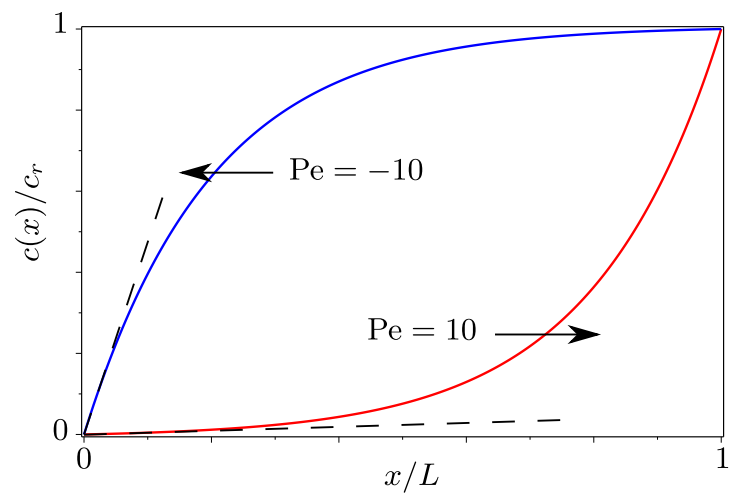

FIG. 2. The analytical concentration profile of Eq. (19) for the limiting current condition $c_{l}=0$ for a flow to the left with $\mathrm{Pe}=-10$ and a flow to the right with $\mathrm{Pe}=10$. The dashed lines indicate the local concentration gradient at $x=0$, illustrating how a flow to the left strongly increases the limiting current while a flow to the right reduces it. A limiting current with $c_{l}=0$ can arise for an anion flux $N_{-}<0$ and $M_{+}=0$ or a cation flux $N_{+}<0$ and $N_{-}=0$ resulting in a positive, or negative limiting current, respectively, in agreement with Eq. (B2).

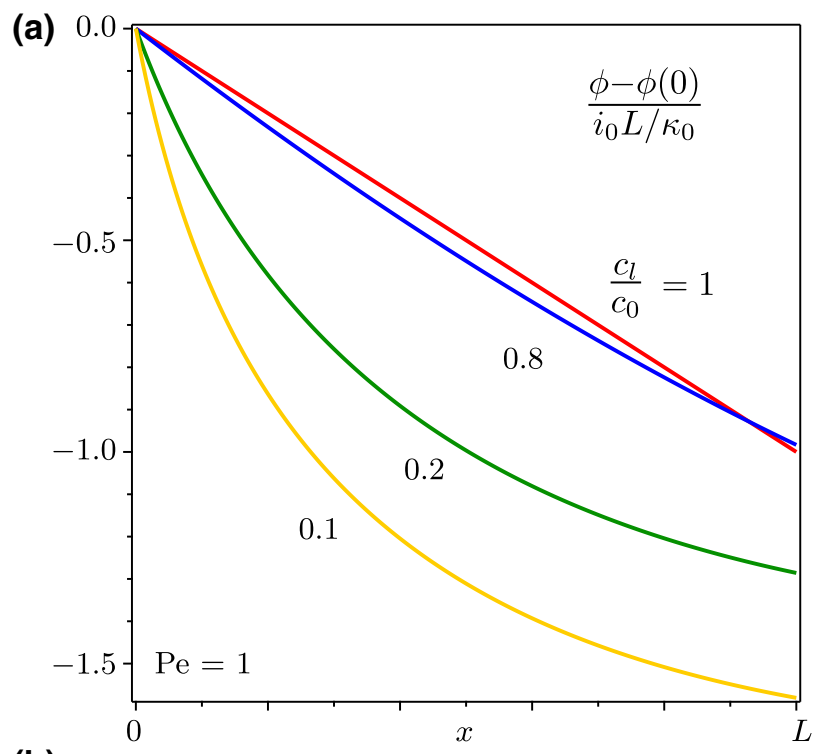

(b)

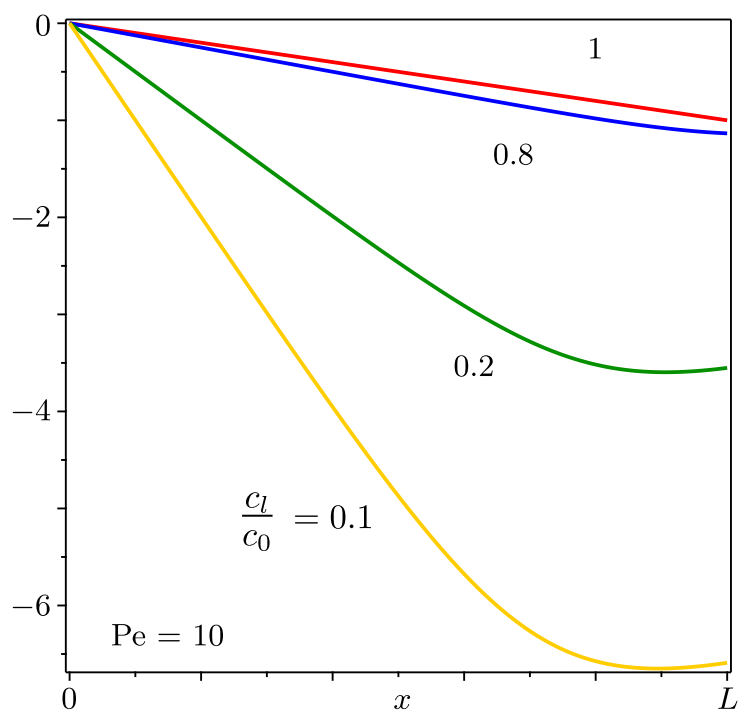

FIG. 3. The potential $\phi(x)-\phi(0)$ nondimensionalized with the characteristic ohmic voltage $i_{0} L / \kappa_{0}=4 t_{-}(R T / F)$ for a current density $i_{0} \equiv 4 F D_{-} c_{0} / L$ and various different concentrations $c_{l}$ and Péclet numbers, $\mathrm{Pe}=1$ (a) and $\mathrm{Pe}=10$ (b). The average concentration of the left and right electrolytes $\left(c_{l}+c_{r}\right) / 2=c_{0}$ is fixed. We use $\bar{\chi} \equiv \chi /\left(i_{0} L / \kappa_{0}\right)=\left(D_{+}-D_{-}\right) / 4 D_{-} \approx-0.16$ representative for potassium hydroxide, $\mathrm{KOH}$. Because $\chi<0$, the diffusion potential, the first term of Eq. (22), is positive. This is why, for $\mathrm{Pe}=1$ (left), the potential difference $\Delta \phi=\phi(L)-$ $\phi(0)$ is smaller for $c_{l} / c_{0}=0.8$ compared to when $c_{l}=c_{0}$. It is also the reason for the nonmonotonous behavior of the potential near $x=L$ when $\mathrm{Pe}=10$ (right).

as a logarithmic part. We introduced the characteristic "conductivity"

$$
\kappa_{\mathrm{c}} \equiv \frac{N}{u c} \kappa=\frac{\kappa_{l} e^{\mathrm{Pe}}-\kappa_{r}}{e^{\mathrm{Pe}}-1} \approx \begin{cases}\kappa_{l}, & \mathrm{Pe} \gg 1 \\ \kappa_{r}, & \mathrm{Pe} \ll-1\end{cases}
$$


We note from Eq. (12) that $u c / N$ is the fraction of the salt flux carried by advection. When $|\mathrm{Pe}| \gg 1$, the final logarithmic term in Eq. (22) can usually be neglected, and $\kappa_{c}$ represents the upstream conductivity. Equation (22) gives the potential drop $\Delta \phi \equiv \phi(L)-\phi(0)$ in terms of the concentrations $c_{l}$ and $c_{r}$ and the current density $i$ as

$$
\Delta \phi=-\chi \ln \left(\frac{c_{r}}{c_{l}}\right)-\frac{i L}{\kappa_{\mathrm{c}}}\left(1-\frac{\ln \left(c_{r} / c_{l}\right)}{\mathrm{Pe}}\right) .
$$

When $c_{l}=c_{r}=c_{0}$, this immediately reduces to the expected ohmic drop

$$
\Delta \phi=-\frac{i L}{\kappa_{0}} .
$$

In general, Eq. (24) also includes the diffusion potential proportional to $\chi$ and takes into account the impact of flow on migration through the concentration profile. Surprisingly, this simple expression for the potential drop in the presence of diffusion, migration, and advection does not seem to have been reported previously. See however Appendix $\mathrm{C}$ for various limiting cases and their applications that can be found in the literature. For example, in Appendix B we discuss the case in which one of the ions is nonmobile, which is relevant near an ion-exchange membrane or when only one of the ions participates in a reaction. In Ref. [31], Eq. (24) was derived for the case of a monovalent electrolyte. A well-known result is obtained in the no-flow limit $\mathrm{Pe} \rightarrow 0$. In this case $\kappa_{\mathrm{c}} \rightarrow\left(\kappa_{l}-\kappa_{r}\right) / \mathrm{Pe}$ so that Eq. (24) becomes

$$
\Delta \phi=\left(\chi-\frac{i L}{\kappa_{l}-\kappa_{r}}\right) \ln \left(\frac{c_{l}}{c_{r}}\right) \quad(\mathrm{Pe}=0) .
$$

When additionally one of the ions is nonmobile, the often encountered Eqs. (B4), (B5), (B7), and (B8) of Appendix B 2 result.

\section{Quasisteady state model \\ 1. Governing equations}

Equation (24) for $\Delta \phi$, in combination with Eq. (6) for $u$, can be solved self-consistently when a relation for $c_{r} / c_{l}$ is provided. For this purpose, we consider the configuration of Fig. 4, in which two well-mixed volumes $V_{l}$ and $V_{r}$ are connected to the porous layer on the left and right, respectively. In these volumes, we assume that a redox reaction takes place in which only one of the two ion species of the binary electrolyte participates. In steady state, by conservation of charge, the amount of this reactive ion consumed in one of the volumes must be generated in the other. We neglect the amount of ions in the volume of the porous layer, or separator. Consistent with this assumption, we $d o$ include the relatively slow time variation in the concentrations $c_{l}$ and $c_{r}$, but at the same time we assume that the

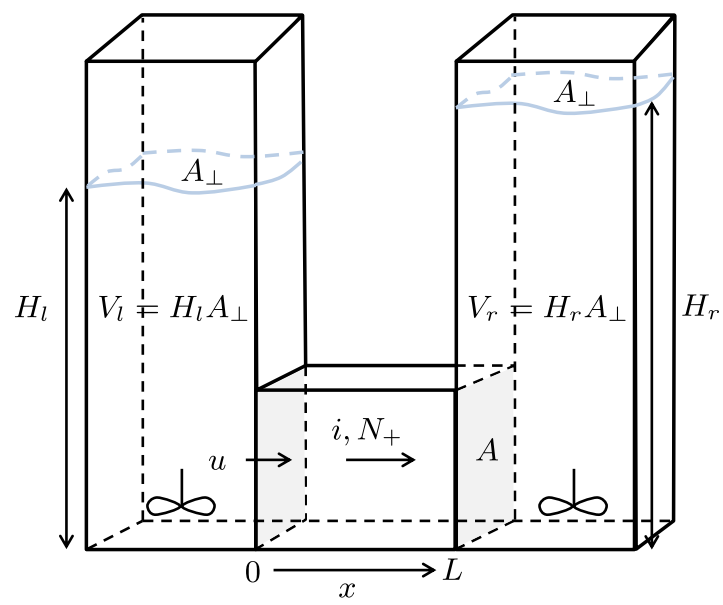

FIG. 4. A schematic illustration of the experimental setup and the configuration to which the analysis of Sec. II D refers. The indicated direction of the constant velocity $u$, current density $i$, and cation flux $N_{+}$are consistent with the experiments of Sec. III, when the anode and cathode are on the left and right, respectively. The direction of these fluxes can be chosen arbitrarily in the analysis. The left and right volumes are well stirred, in contrast with the unstirred layer between $x=0$ and $x=L$.

steady-state equations of the previous section hold in the thin separator. With an index $i=n$ to denote the nonreacting ion, the conservation equation for the nonreacting ion concentration $c_{n}$ in the left volume reads

$$
\frac{d\left(v_{n} c_{l} V_{l}\right)}{d t}=-N_{n} A
$$

The continuity equation for the left liquid volume itself reads

$$
\frac{d V_{l}}{d t}=-u A
$$

Inserting Eqs. (15) and (21) into Eq. (27), using Eq. (28), leads to the time-evolution equation

$$
\frac{d c_{l}}{d t}=\frac{c_{0}\left(1-i / i_{\mathrm{lim}}\right)-c_{l}}{\tau}
$$

where $c_{0} \equiv\left(V_{l} c_{l}+V_{r} c_{r}\right) / 2 V_{0}$ and $V_{0} \equiv\left(V_{l}+V_{r}\right) / 2$ are the average electrolyte concentration and vessel volume, respectively. The limiting current density $i_{\text {lim }}=V_{l} c_{0} / A t_{n} \tau$, where

$$
\begin{array}{r}
\tau=\frac{e^{\mathrm{Pe}}-1}{\mathrm{Pe}} \frac{V_{l} V_{r}}{V_{0}^{2}} \tau_{0} \quad \text { with } \tau_{0} \equiv \frac{L V_{0}}{2 A D_{a}}, \\
i_{\lim }=\frac{\mathrm{Pe}}{e^{\mathrm{Pe}}-1} \frac{V_{0}}{V_{r}} i_{0} \quad \text { with } i_{0} \equiv z_{n} v_{n} \frac{2 F D_{a} c_{0}}{L t_{n}} .
\end{array}
$$


Assuming that $\Delta p=0$, we can use Eq. (6) in Eq. (28) to give

$$
2 \frac{\tau_{0}}{V_{0}} \frac{d V_{l}}{d t}=-\mathrm{Pe}=\frac{\mathcal{M}}{D_{a}} \Delta \phi .
$$

A complete model describing the time evolution consists of the two ordinary differential equations, Eqs. (29) and (32), combined with Eq. (24) for $\Delta \phi$. Since a steady state is assumed in deriving Eq. (24), these equations only describe large timescales compared to the diffusive timescale of the separator. Aguilella et al. [35] considered, for a monovalent binary electrolyte and a constant velocity, the analytical series solution to the full transient Nernst-Planck equations. In Appendix E we consider several analytical solutions to the much simpler evolution equation (29).

To obtain a full electrochemical cell characterization, we can add one of the reaction models discussed in Appendix F. For Tafel kinetics, with a reaction on the left electrode that is first order in the electrolyte concentration $c_{l}$, Eq. (F3) gives

$$
E_{\text {cell }}-E_{\mathrm{oc}}=\overbrace{-\Delta \phi}^{\text {separator }}+\overbrace{b \ln \left(\frac{i}{\mathcal{E} i_{*}}\right)}^{\text {activation }}+\overbrace{b_{l} \ln \left(\frac{c_{0}}{c_{l}}\right)}^{\text {concentration }} .
$$

The voltage losses on the right-hand side represent the separator potential drop, the combined anodic and cathodic activation overpotentials, and the concentration overpotential. Here the combined Tafel slope $b=b_{l}+b_{r}$ is the sum of that of the left and right electrode reactions, and the exchange current density $i_{*}$ also includes contributions from both reactions. The electrode effectiveness factor $\mathcal{E} \leq$ 1 accounts for the reaction taking place inhomogeneously over the width of the electrode [36-38].

\section{Limiting current density}

When $c_{l}=0$, we have $2 V_{0} c_{0} / V_{r}=c_{r}$ and Eq. (31) for $i_{\text {lim }}$ agrees with Eq. (B2) derived in Ref. [6]. For a monovalent binary electrolyte with $z_{+}=-z_{-}=v_{+}=v_{-}=1$, we recover the equations of Ref. [31].

From Eq. (31), initially when $V_{r}=V_{0}$, we have

$$
\frac{i_{\lim }}{i_{0}}=\frac{\mathrm{Pe}}{e^{\mathrm{Pe}}-1} \approx \begin{cases}\mathrm{Pe} / e^{\mathrm{Pe}} \approx 0, & \mathrm{Pe} \gg 1, \\ 1-\mathrm{Pe} / 2, & |\mathrm{Pe}| \ll 1, \\ -\mathrm{Pe}, & \mathrm{Pe} \ll-1 .\end{cases}
$$

The factor $\mathrm{Pe} /\left(e^{\mathrm{Pe}}-1\right)$ derives from Eq. (21) that, for $c_{l}=0$, gives the salt flux as $N=-\left[\mathrm{Pe} /\left(e^{\mathrm{Pe}}-1\right)\right]\left(D c_{r} / L\right)$. It shows how a flow to the right, $\mathrm{Pe}>0$, opposes the diffusive flux $N=-D c_{r} / L$ while a flow to the left, $\mathrm{Pe}>0$, enhances it. From Eq. (30), it follows that the associated timescale $\tau$ is divided by the same factor.

\section{Dimensionless numbers}

The solution of Eqs. (29), (32), and (24) can be expressed in terms of the dimensionless quantities $c_{l} / c_{0}$, $\Delta \phi / \chi$, and $V_{l} / V_{0}$ as a function of the dimensionless time $t / \tau_{0}$ or $t / \tau(0)$. Using the ohmic drop $i_{0} L / \kappa_{0}$ as a characteristic potential, the relevant free model parameters can then be expressed in terms of the dimensionless numbers

$$
\bar{\chi} \equiv \frac{\chi}{i_{0} L / \kappa_{0}}, \quad \mathrm{Pe}_{0} \equiv \frac{i_{0} L / \kappa_{0}}{D_{a} / \mathcal{M}}, \quad \frac{i}{i_{0}} .
$$

Here $\bar{\chi}$ and $\mathrm{Pe}_{0}$ are approximately constant material parameters, while $i / i_{0}$ is an operational parameter. Using Eqs. (18), (31), (8), and (11) we can write

$$
\frac{i_{0} L}{\kappa_{0}}=\frac{2 D_{a}}{z_{n} D_{n}} \frac{R T}{F} .
$$

With Eqs. (17) and (13), this gives $\bar{\chi}=\left[z_{n} D_{n} /\left(z_{+}-z_{-}\right)\right]$ $\left[\left(D_{+}-D_{-}\right) / 2 D_{+} D_{-}\right]$. This dimensionless number is typically small so that, for $i=i_{0}$, the ohmic potential drop dominates over the diffusion potential. For potassium hydroxide, for example, $\bar{\chi}=\left(D_{+}-D_{-}\right) / 4 D_{-} \approx-0.16$.

With Eq. (36) we can write $\mathrm{Pe}_{0}=\left(2 \mathcal{M} / z_{n} D_{n}\right)(R T / F)$. Assuming that at $t=0$ there are no concentration gradients, Eq. (24) gives $\Delta \phi=-i L / \kappa_{0}$. Inserting in Eq. (32) gives $\mathrm{Pe}(t=0)=\left(i / i_{0}\right) \mathrm{Pe}_{0}$, so that $\mathrm{Pe}_{0}$ is the initial Péclet number when $i=i_{0}$.

In general, $c_{l}(0) / c_{0}$ and $V_{l}(0) / V_{0}$ are two further dimensionless numbers characterizing the system in case of unequal starting concentrations or volumes, respectively.

\section{Draining time}

In the case of a constant flow velocity $u$ and $V_{l}(0)=$ $V_{r}(0)=V_{0}$ it will take a time

$$
t_{0}=\frac{V_{0}}{|u| A}=\frac{2 \tau(0)}{\left|e^{\mathrm{Pe}}-1\right|}
$$

to fully drain the left (when $u>0$ ) or right $(u<0)$ electrolyte volume. When $V_{l}$ or $V_{r}$ approaches zero, we see from Eq. (30) that the equilibration time $\tau$ also decreases. From Eq. (29), this speeds up the approach to the limiting condition $c_{l}=0$ (when $i / i_{\lim }>1$ ) or $c_{r}=0$ (when $\left.i / i_{\lim }<-1\right)$. A limiting condition $\left(i / i_{\lim }>1\right)$ or a steady state $\left(i / i_{\lim }<1\right)$ is reached well before draining only if $\tau(0) \ll t_{0}$, which requires Pe to be positive but well below 1 . When Pe is large and negative, we have $t_{0} \approx 2 \tau(0)$ and draining and equilibration always go hand in hand.

\section{E. Numerical time-dependent solutions}

In this section we show numerical solutions to Eqs. (29), (32), and (24). This system of ordinary differential equations constitutes an initial value problem, for which we use 
TABLE I. Approximate experimental parameter values at different $\mathrm{KOH}$ concentrations $c_{0}$ at $25^{\circ} \mathrm{C}$. Here $D_{a}$ from Ref. [39] and $\kappa_{0}$ from Ref. [40] are corrected with $\epsilon / \tau \approx 0.31$. Furthermore, we use $L=0.5 \mathrm{~mm}, V_{l}(0)=V_{r}(0)=V_{0}=24 \mathrm{ml}$, a constant $t_{+}=0.27$, and $\chi=-12 \mathrm{mV}$. The effective electrical mobility $\mathcal{M}$ is fitted from Fig. 12 and the electro-osmotic flow mobility $\mathcal{M}^{\text {flow }}$ is calculated from Eq. (42).

\begin{tabular}{lccccc}
\hline \hline$c_{0}$ & $M$ & 0.1 & 1 & 2.9 & 5.7 \\
$D_{a}$ & $10^{-10} \mathrm{~m}^{2} / \mathrm{s}$ & 8.8 & 8.8 & 10 & 12 \\
$\kappa_{0}$ & $\mathrm{~S} / \mathrm{m}$ & 0.84 & 6.6 & 15 & 19 \\
$i_{0}=2 F D_{a} c_{0} / L t_{+}$ & $\mathrm{kA} / \mathrm{m}^{2}$ & 0.13 & 1.3 & 4.3 & 9.4 \\
$\tau_{0}=L V_{0} / 2 A D_{a}$ & $10^{3} \mathrm{~s}$ & 6.6 & 6.7 & 5.7 & 5.1 \\
$l_{e}$ & $\mathrm{~mm}$ & 0.3 & 0.5 & 0.7 & 0.6 \\
$\mathcal{M}$ & $10^{-9} \mathrm{~m}^{2} / \mathrm{s} \mathrm{V}$ & 6.7 & 3.6 & 3.1 & 2.6 \\
$\mathcal{M}^{\text {flow }}$ & $10^{-9} \mathrm{~m}^{2} / \mathrm{s} \mathrm{V}$ & 7.0 & 6.6 & 9.7 & 12 \\
\hline \hline
\end{tabular}

as initial conditions $c_{l}=c_{r}=c_{0}$ and $V_{l}=V_{r}=V_{0}$. The default fifth-order accurate Runge-Kutta-Fehlberg method in MAPLE $^{\circledR}$ is used for the time integration.

We consider a flow from left to right, with an initial Péclet number $\operatorname{Pe}(0)=\ln (2) \approx 0.69$, a constant $i / i_{0}=$ 1.221 , and $\bar{\chi}=-0.16$. This corresponds more or less to the experiments with $c_{0}=0.1 \mathrm{M}$, detailed in the next section and Tables I and II. For a comparison with and verification of the numerical results, the analytical solution to Eq. (29) for a constant $\mathrm{Pe}=\ln 2$, Eq. (E8), is also shown using dashed lines.

For small times $t \ll \tau_{0}$, the numerical and analytical solutions agree, and both show how the flow, from left to right, decreases $V_{l}$. The concentration $c_{l}$ decreases as a consequence of our choice for a positive $i / i_{0}$. This indicates, for example, a current density from left to right, $i>0$, in the case of a nonreacting cation $z_{n}>0$. In this case the reacting anions will diffuse to the left, causing $c_{l}$ to decrease with time.

Because $\bar{\chi}<0$ and $c_{r} / c_{l}>1$, the first term of Eq. (24) provides a negative contribution $\bar{\chi} \ln \left(c_{r} / c_{l}\right)$ to $-\Delta \phi /\left(i_{0} L / \kappa_{0}\right)$. Initially, for $0<t / \tau_{0} \leq 0.1$, this causes $-\Delta \phi /\left(i_{0} L / \kappa_{0}\right)$ to decrease, as is visible from the very slight dip in Fig. 5. As $c_{l}$ decreases further, however, the decrease in $\kappa_{c}$ of Eq. (23) causes the second ohmic term in Eq. (24) to dominate and increase $-\Delta \phi /\left(i_{0} L / \kappa_{0}\right)$ again. Beyond $t / \tau_{0} \geq 0.4$, this increase starts to noticeably drive more electro-osmotic flow, so that the numerical solution

TABLE II. Modeling parameters characteristic for $t=0$ at a current density $i=i_{0}$ using the same conditions as in Table I.

\begin{tabular}{lccccc}
\hline \hline$c_{0}$ & $M$ & 0.1 & 1 & 2.9 & 5.7 \\
$\mathrm{Pe}=\mathrm{Pe}_{0}=\mathcal{M} i_{0} L / \kappa_{0} D_{a}$ & $\ldots$ & 0.6 & 0.4 & 0.4 & 0.6 \\
$\mathrm{Pe} /\left(e^{\mathrm{Pe}}-1\right)$ & $\ldots$ & 0.7 & 0.8 & 0.8 & 0.7 \\
$\tau=\left(e^{\mathrm{Pe}}-1\right) \tau_{0} / \mathrm{Pe}$ & $10^{3} \mathrm{~s}$ & 8.9 & 8.2 & 7.1 & 6.8 \\
$i_{\lim }=i_{0} \mathrm{Pe} /\left(e^{\mathrm{Pe}}-1\right)$ & $\mathrm{kA} / \mathrm{m}^{2}$ & 0.1 & 1 & 3 & 7 \\
$-\bar{\chi} \equiv-\chi /\left(i_{0} L / \kappa_{0}\right)$ & $\%$ & 16 & 13 & 8.2 & 4.8 \\
\hline \hline
\end{tabular}

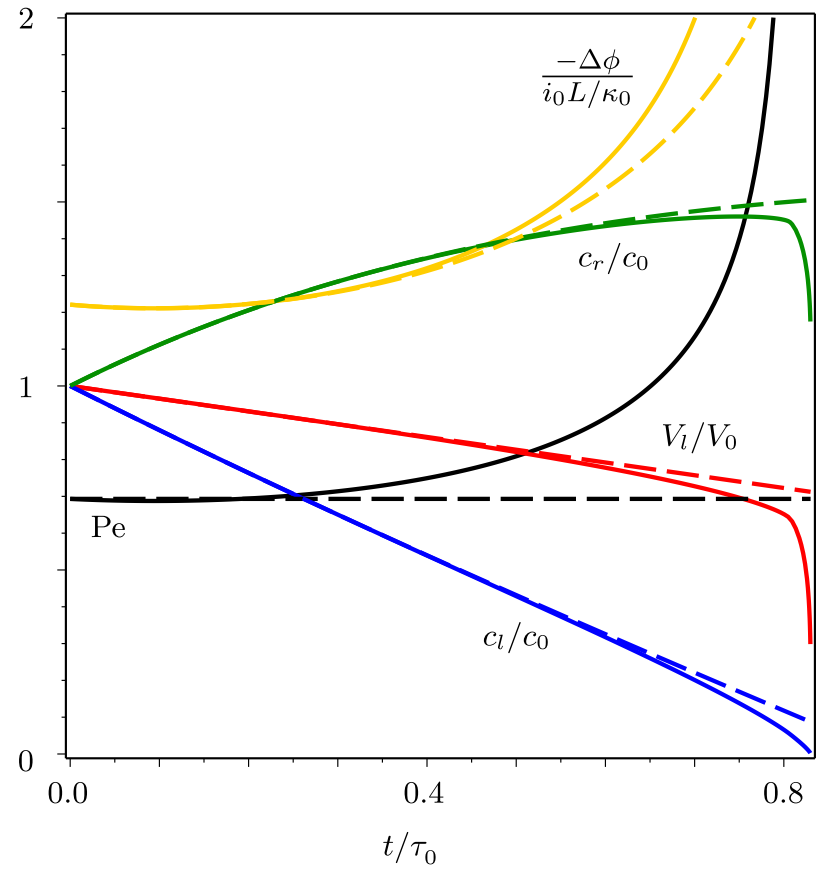

FIG. 5. Various dimensionless quantities as a function of the dimensionless time $t / \tau_{0}$. The numerical solution of Eqs. (28)-(32) and (22) (solid lines) is compared to the analytical solution of Eq. (E8) (dashed) for constant $\mathrm{Pe}=\ln 2 \approx 0.7, i / i_{0}=$ 1.221, and $\bar{\chi}=-0.0118$. As initial conditions at $t=0$, we use $c_{l}=c_{r}=c_{0}$ and $V_{l}=V_{r}=V_{0}$. These conditions are similar to the experiments performed at $c_{0}=0.1 M$ detailed in Tables I and II. In Fig. 6 we show the corresponding simulated fluxes.

deviating noticeably from the constant-flow velocity analytical solution. The enhanced flow velocity accelerates the draining of $V_{l}$. Finally, when $t / \tau_{0} \approx 0.83$, a limiting condition is reached when $c_{l}$ vanishes. Note that this happens well before the left volume would otherwise have been drained. As a result of the diverging electroosmotic flow velocity, however, in a short time the entire low-concentration content of the left volume enters the right volume, where it causes the concentration to drop significantly.

In Fig. 6 we plot the simulated molar fluxes of Fig. 5, nondimensionalized with the characteristic flux $i_{0} / F$. To relate to the experiments of Sec. III, we consider a monovalent electrolyte with $\left|z_{ \pm}\right|=v_{ \pm}=1$ and a nonreacting cation so that $z_{n}=z_{+}=1$. In this case, from Eqs. (15) and (21),

$$
\begin{gathered}
\frac{F N_{ \pm}}{i_{0}}=\frac{F N}{i_{0}} \pm t_{ \pm} \frac{i}{i_{0}}, \\
\frac{F N}{i_{0}}=\frac{t_{+}}{2} \frac{\mathrm{Pe}}{e^{\mathrm{Pe}}-1} \frac{c_{l} e^{\mathrm{Pe}}-c_{r}}{c_{0}},
\end{gathered}
$$

where the same diffusion-enhancement factor $\mathrm{Pe} /\left(e^{\mathrm{Pe}}-1\right)$ of Eq. (34) appears again. Initially, $N / i_{0}$ is positive due 


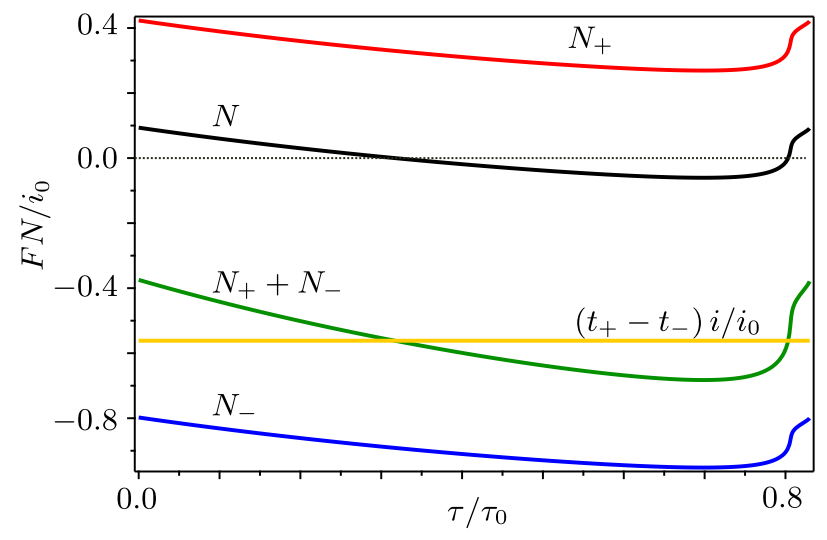

FIG. 6. Various fluxes, nondimensionalized with $i_{0} / F=$ $2 F D_{a} c_{0} / L t_{+}$, corresponding to the numerical model results of Fig. 5 for $\operatorname{Pe}(0)=\ln (2) \approx 0.7$ and $i / i_{0}=1.221$ using $\left|z_{ \pm}\right|=$ $v_{ \pm}=1$ and $t_{+}=0.23$ and $t_{-}=0.73$ appropriate for $\mathrm{KOH}$.

to the positive advective flux from left to right. As time progresses, a concentration difference $c_{l}-c_{r}<0$ drives an opposing diffusive flux so that, when $c_{l}=c_{r} e^{-\mathrm{Pe}}$, the advective and diffusive fluxes cancel, and $N=0$. From Fig. 6, this happens around $t / \tau_{0} \approx 0.3$. As the flow velocity increases dramatically as the limiting current is approached, for $t / \tau_{0} \gtrsim 0.8$, the advective flux makes $N / i_{0}$ positive again.

Because the diffusive and advective fluxes so nearly oppose each other in this particular case, the species fluxes $N_{i}$ are primarily determined by the final term in Eq. (38), representing migration. Therefore, for $i / i_{0}>0$, the nonreacting cations move to the right in the direction of the electric field, while the reacting anions move in the opposite direction.

\section{EXPERIMENTS}

To validate the theory developed in the previous sections, we consider the electro-osmotic flow through a porous diaphragm driven by a potential difference created by two electrodes placed directly adjacent. This "zero-gap" configuration is common in modern alkaline water electrolysers for the production of hydrogen. In Ref. [31] we reported that a flow can be established from anode to cathode, lowering the limiting current and impacting crossover of dissolved gases. Here we consider in more detail these, and additional, experimental results. The experiments discussed here also include concentrated electrolytes, which will introduce deviations from the dilute solution theory considered above.

\section{A. Experimental setup}

The experimental setup shown in Fig. 7 is constructed from several layers of plexiglass as shown in Fig. 8. The left and right volumes are each filled with $V_{l}=V_{r}=24$

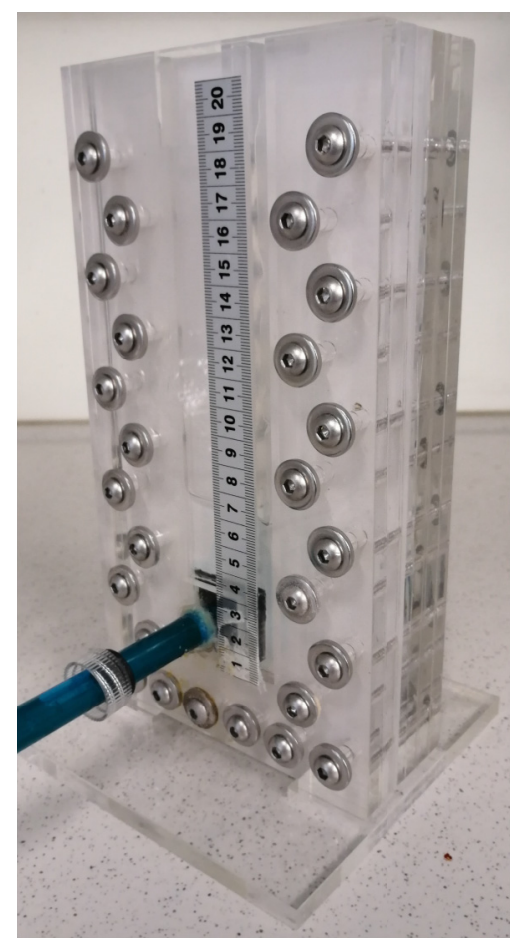

FIG. 7. The experimental setup with the channel through which the evolved gases leave, and the smaller capillary for measuring the level, to the left and right of the measurement tape, respectively. See also the highlighted regions in Fig. 8. Between 0 and $3 \mathrm{~cm}$, the black square electrode is visible as well as the $p \mathrm{H}$ sensor sticking out of the setup. On each side of the white Zirfon-Perl separator are four layers of polymethyl methacrylate (plexiglas) laser cut as shown in Fig. 8.

$\mathrm{ml}$ of an aqueous potassium hydroxide solution, separated by Agfa's Zirfon Perl UTP 500. This separator is based on 85 weight percent (wt \%) $\mathrm{ZrO}_{2}$ particles and $15 \mathrm{wt} \%$ polysulfone backbone and has an average pore size of $0.15 \mu \mathrm{m}$, a porosity of $\epsilon \approx 0.5$, and a thickness of $L \approx 0.5 \mathrm{~mm}$. Roughly equally thick expanded metal

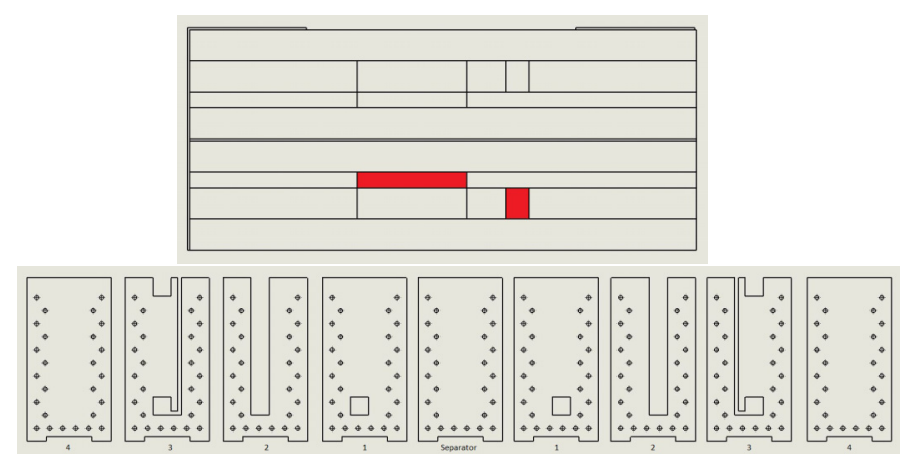

FIG. 8. The various laser-cut plexiglass layers making up the experimental setup of Fig. 7 in plan view (top), with the rectangular gas-evolution exit and nearly square level measurement capillary highlighted in red, and in enlarged view (bottom). 
(a)

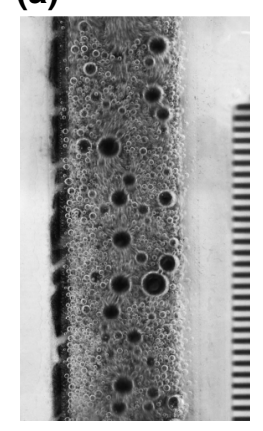

(b)

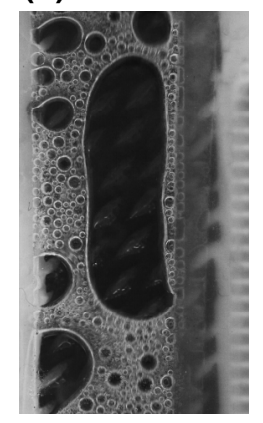

(c)

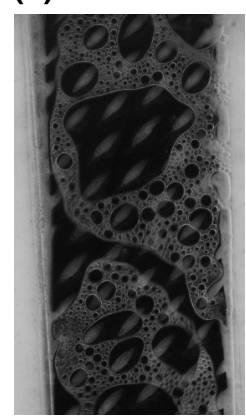

FIG. 9. The same electrode material as used in the experiments placed in a configuration where the transparent back wall is positioned at a distance of $2 \mathrm{~mm}$ (a), $1.3 \mathrm{~mm}$ (b), or $0.5 \mathrm{~mm}$ (c) to provide better optical access to the cathode and hydrogen bubbles in this case. These experiments are performed with $30 \mathrm{wt} \%$ $\mathrm{KOH}$ and at $E_{\text {cell }}=2.2 \mathrm{~V}$. The black and white ruler indicates a millimeter scale.

Permascand electrodes of $A=32 \times 32 \mathrm{~mm}^{2} \approx 10 \mathrm{~cm}^{2}$ are pressed to the separator at their edges and connected to a power source. See Fig. 7 for their position in the setup and Fig. 9 for an enlargement of the same electrodes in a different setup. Parallel to the $32 \times 2 \mathrm{~mm}^{2}$ gas outlets shown in Fig. $8,6 \times 8 \mathrm{~mm}^{2}$ bubble-free channels are used to determine the liquid level. Calibration showed a combined cross-sectional area of $A_{\perp}=117 \mathrm{~mm}^{2}$. A BK Precision PV9100 power source is used in constant current mode, which has an accuracy less than or equal to $0.02 \%+2 \mathrm{mV}$ and less than or equal to $0.1 \%+15 \mathrm{~mA}$. The electrolyte

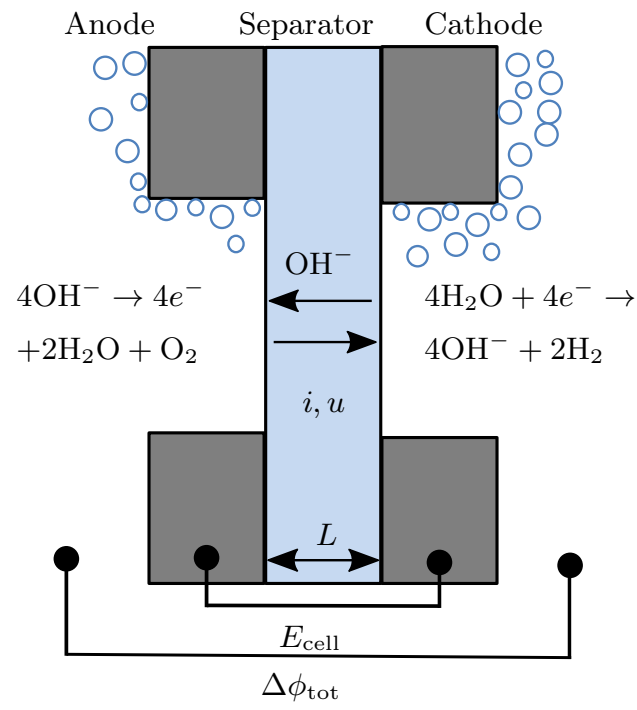

FIG. 10. A schematic repeating unit of the zero-gap extended mesh electrode-separator region in the alkaline water electrolysis experiments of Sec. III. The connection points for electrode and electolyte voltages $E_{\text {cell }}$ and $\Delta \phi_{\text {tot }}$, respectively, are indicated with connected filled circles. The current $i$ and electro osmotic velocity $u$ are directed from anode to cathode.

voltage drop $\Delta \phi_{\text {tot }}$, indicated in Fig. 10, is recorded using a Madgetech RFVolt2000A voltmeter with an accuracy of $0.05 \%$.

\section{B. Parameters}

At dilute concentrations and $25^{\circ} \mathrm{C}$, the diffusivities of $\mathrm{OH}^{-}$and $\mathrm{K}^{+}$are 1.96 and $5.30 \times 10^{-9} \mathrm{~m}^{2} / \mathrm{s}$ [32], respectively. This gives transference numbers of $t_{+}=0.27$ and $t_{-}=0.73$, using Eq. (11), and a molecular ambipolar diffusivity of $2.9 \times 10^{-9} \mathrm{~m}^{2} / \mathrm{s}$, using Eq. (13). We correct according to Eq. (7), using the separator porosity $\epsilon \approx 0.5$ and tortuosity $\tau=1.62$. The latter value has a significant uncertainty and also depends on the age of the separator [29]. In the dilute limit this gives $D_{a} \approx 0.9 \times 10^{-9} \mathrm{~m}^{2} / \mathrm{s}$. Data of measured salt diffusivities [39] listed in Table I indicate that $D_{a}$ first slightly decreases before increasing again with increasing electrolyte concentration. At $5.5 \mathrm{M}$ the diffusivity increases by about $30 \%$ compared to its value at infinite dilution. We additionally note that the temperature is not controlled so that, as the electrolyte heats up during the experiment, the diffusivity may also increase.

For the molecular conductivity of the unconfined electrolyte, we use the empirical formula $\kappa_{\infty}=-204.1 c-$ $0.28 c^{2}+0.5332 c T+20720 c / T+0.1043 c^{3}-0.00003 c^{2}$ $T^{2}$ of Ref. [40], in which $c$ is expressed in $M$ and we use $T=298 \mathrm{~K}$. For the used concentrations of $c_{0}=0.1 M, 1 M$, $2.9 M$, and $5.5 M$, this gives values of $2.7,21,48$, and 62 $\mathrm{S} / \mathrm{m}$, respectively. To obtain effective porous medium values, we use the same correction as for the diffusivity to give $\kappa_{0}=\kappa_{\infty} \epsilon / \tau$ reported in Table I.

In Table II we list the dimensionless numbers of Eq. (35). The characteristic ratio $\bar{\chi} \equiv \chi /\left(i_{0} L / \kappa_{0}\right)$ of the diffusion potential and ohmic drop shows that, while the ohmic will always dominate, the effect of the diffusion potential is largest at the lower concentration. The reason is that the conductivity increases sublinearly with concentration at higher concentrations.

\section{Measurements}

A given fixed current is switched on at $t=0$ while we record, in time, the cell potential $E_{\text {cell }}$, the potential difference $\Delta \phi_{\text {tot }}$ between the left and right electrolytes, the $p \mathrm{H}$ in the anolyte, and the variation in the levels of the left and right electrolytes. In the next sections these measurements will be discussed in more detail.

\section{Electro-osmotic velocity $\boldsymbol{u}$}

The observed flow velocity is from the anode on the left to the cathode on the right, so opposite to the expected level change due to water consumption at the cathode and production at the anode. In Fig. 11 we show the difference between the right and left electrolyte levels for various time instances as read off from the measurement tape visible in Fig. 7. Approximately time-averaged values 
(a)
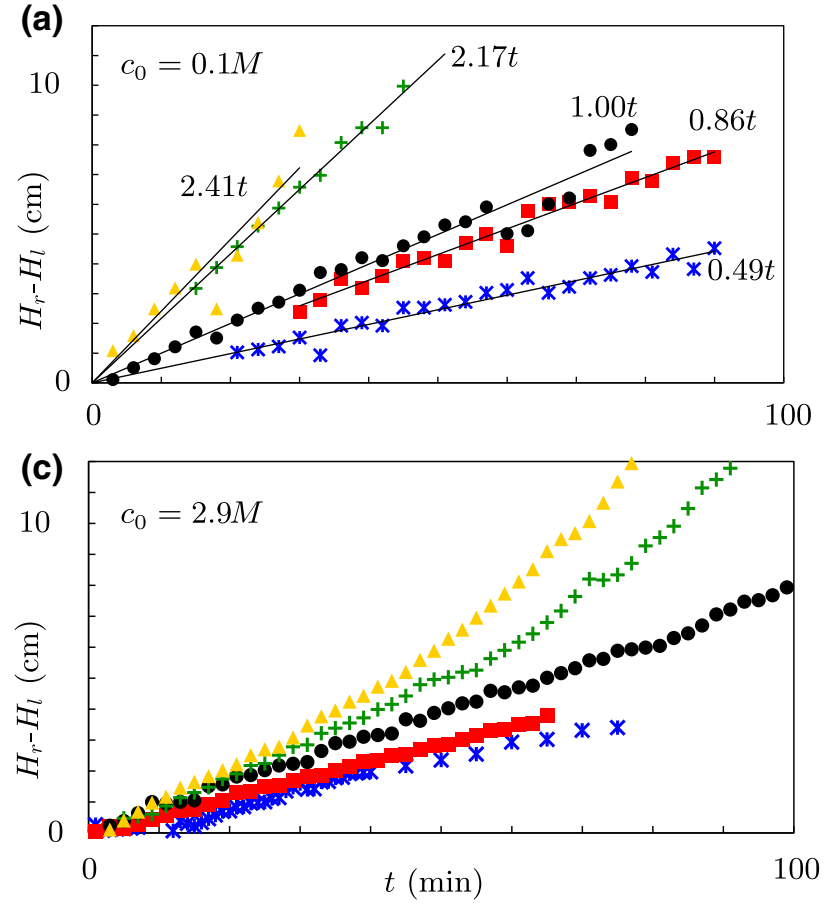

(b)

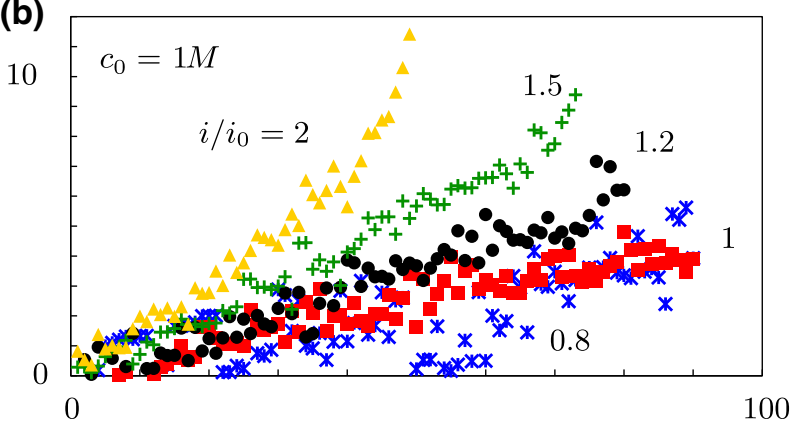

(d)

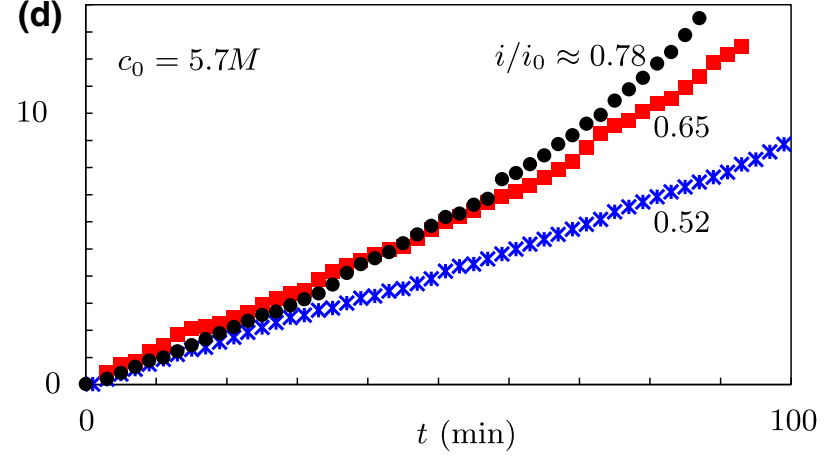

FIG. 11. The recorded differences between the right and left electrolyte level heights, $H_{r}$ and $H_{l}$, respectively. The different colors indicate different current densities $i / i_{0}=0.8$ (blue crosses), 1 (red squares), 1.2 (black circles), 1.5 (green plus signs), and 2 (yellow triangles) as indicated in the top-right figure for $c_{0}=1 \mathrm{M}$. It can be seen that, as the limiting current is approached, the velocity increases. Linear fits of the initial linear phase, as shown for $c_{0}=0.1 M$, are plotted in Fig. 12. The height differences are corrected for water production and consumption through Eq. (40). Note that approximately time-averaged values are recorded except for $c_{0}=1 M$, where we use instantaneous values. This gives an idea of the level fluctuations present.

are recorded, except for the $1 M$ case, where instantaneous values are shown. This gives an idea of the level fluctuations, induced by the unsteady multiphase flow in the hydraulically connected gas outlet channel. From these level differences we calculate the electro-osmotic velocity using

$$
u=\frac{A_{\perp}}{2 A} \frac{d\left(H_{r}-H_{l}\right)}{d t}+\frac{3 i}{2 F V_{m}} .
$$

Here the level heights $H_{r}$ and $H_{l}$ are shown schematically in Fig. 4. The separator area is $A / A_{\perp} \approx 9$ times the area of the combined area of the level and bubble outlet channels. The final term corrects for the water consumed and produced in the reaction. Per two electrons, two molecules of water are consumed at the cathode on the right while one is produced at the anode on the left. Therefore, in the absence of a level rise, a molar flux of $3 i / 2 F$ must exist from anode to cathode, or left to right. The water molar volume in Eq. (40) for all concentrations is around $V_{m} \approx 1.8 \times 10^{-5} \mathrm{~m}^{3} / \mathrm{mol}$.

In Fig. 11 we show that the level difference $H_{r}-H_{l}$ increases at most by $2.4 \mathrm{~mm} / \mathrm{min}$ for $c_{0}=0.1 \mathrm{M}$, or 1.2 $\mathrm{mm} / \mathrm{min}$ per side. According to Eq. (40) this gives a maximum superficial flow velocity $u \approx 0.12 \mathrm{~mm} / \mathrm{min}$ or 2 $\mu \mathrm{m} / \mathrm{s}$. The velocity increases with time as the limiting current is approached, as can be observed from the accelerating level difference for the higher current densities in Fig. 11. This is expected when, as in Eq. (6), the flow is driven by the separator voltage $\Delta \phi$. This voltage increases as the anolyte depletes, when limiting conditions are approached. This is illustrated by the model calculations for Pe in Fig. 5.

As discussed in Sec. II D 4, for modest Péclet numbers, a limiting current can be approached well before one of the electrolyte volumes is drained, in agreement with the observations. The diffusion-enhancement factors $\mathrm{Pe} /\left(e^{\mathrm{Pe}}-1\right) \approx 0.7-0.8$ listed in Table II result in a limiting current roughly $20 \%-30 \%$ lower compared with that in the absence of flow.

In Fig. 12 we show the time rate of change of the level difference, obtained from a linear fit of the initial linear part of the data in Fig. 11. This is plot as a function of the calculated expected separator voltage $-\Delta \phi=i L / \kappa_{0}$, shown as the dashed line in Fig. 13 as a function of $i$. In accordance with Eq. (6), we expect a linear relation between the separator voltage and electro-osmotic flow velocity, which is indeed observed.

\section{Electrolyte concentration}

The electrolyte concentration $c_{0}$ is monitored by measuring the $p \mathrm{H}$ of the anolyte with the sensor clearly visible 

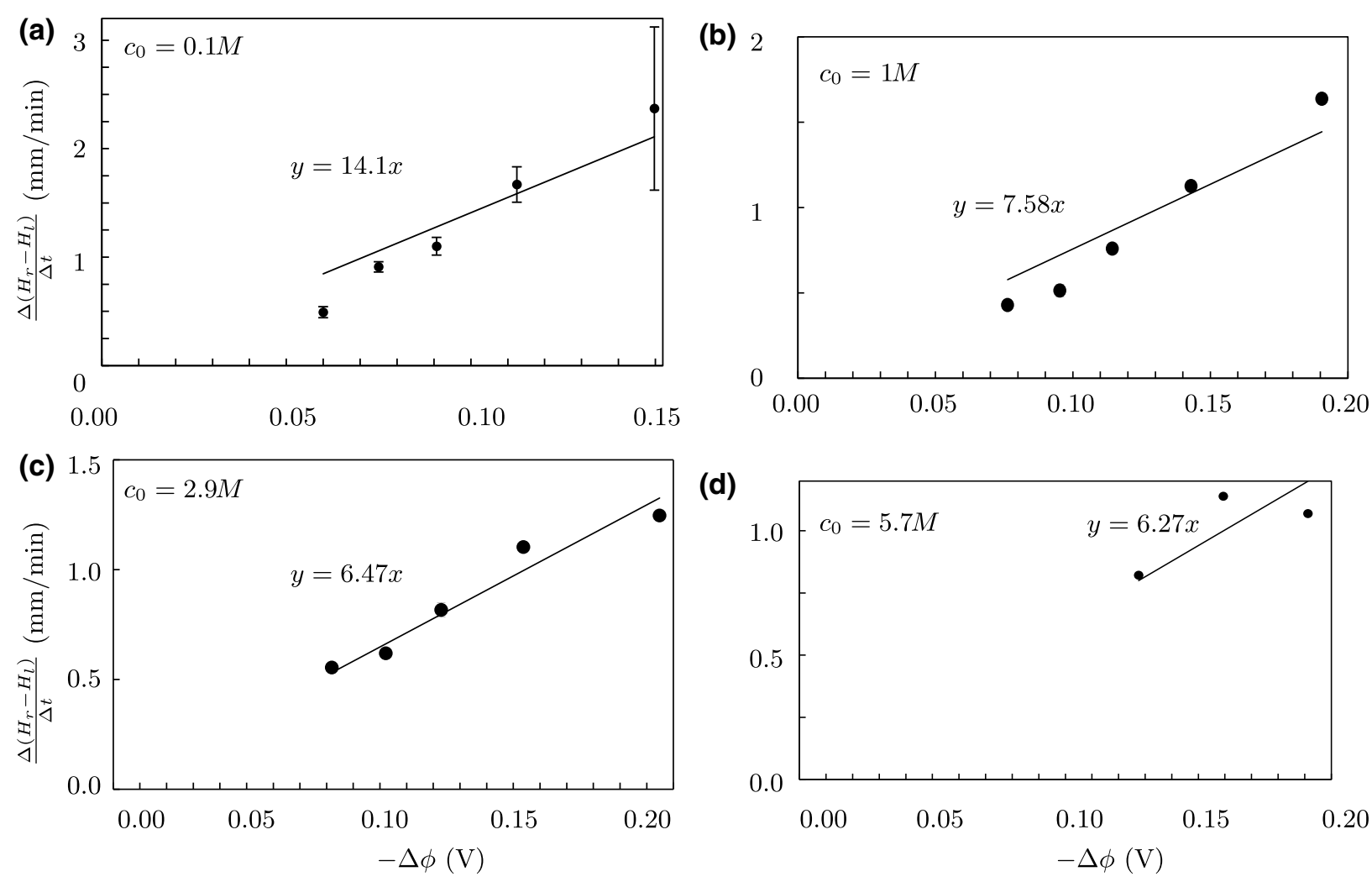

FIG. 12. A linear fit for the time rate of change of the level difference of Fig. 11 plotted as a function of the calculated $-\Delta \phi=i L / \kappa_{0}$, which is shown as the dotted line in Fig. 13. The $95 \%$ confidence interval in the fitted slope is only indicated for $c_{0}=0.1 M$, where it is significant for $i / i_{0}=2$ since only ten data points are used, as shown in Fig. 11. For the other concentrations, the fitting error is generally only a few percent as sufficient measurement points are available for the initially quite linear phase.

in Fig. 7. In Fig. 14 we show the $p \mathrm{H}$ as a function of time for various current densities. For $c_{0}=0.1 \mathrm{M}$ and $1 \mathrm{M}$, the $p \mathrm{H}=14+\log _{10}\left(c_{0}[M]\right)$ should be 13 and 14 , respectively. The initial values shown in Fig. 14 are more or less within the absolute accuracy of 0.1 reported by the supplier of the sensor. The initial linear decrease in $p \mathrm{H}$ indicates an approximately exponentially decreasing concentration in agreement with Eq. (29) near the limiting current density.

\section{Potential drop $\Delta \phi_{\text {tot }}$}

Besides the cell voltage $E_{\text {cell }}$ measured between the two electrodes, we also measure the potential drop over the electrolyte, as illustrated schematically in Fig. 10. Two electrical wires are inserted in the left and right electrolytes, directly behind the electrodes, and connected to a voltmeter. The measured potential difference $-\Delta \phi_{\text {tot }}$ at $t=150 \mathrm{~s}$ after the current is switched on at $t=0$ is shown in Fig. 13. Before this diffusive timescale, there are still significant variations in the potential.

In Fig. 15 we show $-\Delta \phi_{\text {tot }}$ over the duration of an experiment for various concentrations and current densities. For the highest current densities, this potential can be seen to diverge, corresponding to the limiting conditions associated with hydroxide depletion at the anode. As discussed in Sec. II D 4, for modest Péclet numbers, a limiting current can be approached well before one of the electrolyte volumes is drained, in agreement with the observations. The diffusion-enhancement factors $\mathrm{Pe} /\left(e^{\mathrm{Pe}}-1\right) \approx$ $0.7-0.8$ listed in Table II result in a limiting current roughly $20 \%-30 \%$ lower compared to that in the absence of flow.

We also discuss these results in more detail in the next section, where we also make the connection to the theory and simulations of Sec. II.

\section{DISCUSSION}

In Ref. [31] reasonable agreement was found between the numerical solution of Eqs. (28), (29), and (6) and the experimental results for a more or less dilute solution with $c_{0}=0.1 M$. Here we compare numerical and experimental results for higher concentrations more relevant for application in alkaline water electrolysis.

\section{A. Electrode potential drop $i l_{e} / \kappa$}

Besides the measured potential drop $\Delta \phi_{\text {tot }}$, in Fig. 13 we also show the expected potential drop $i L / \kappa$ over the separator, using the parameters of Table I. The approximately 

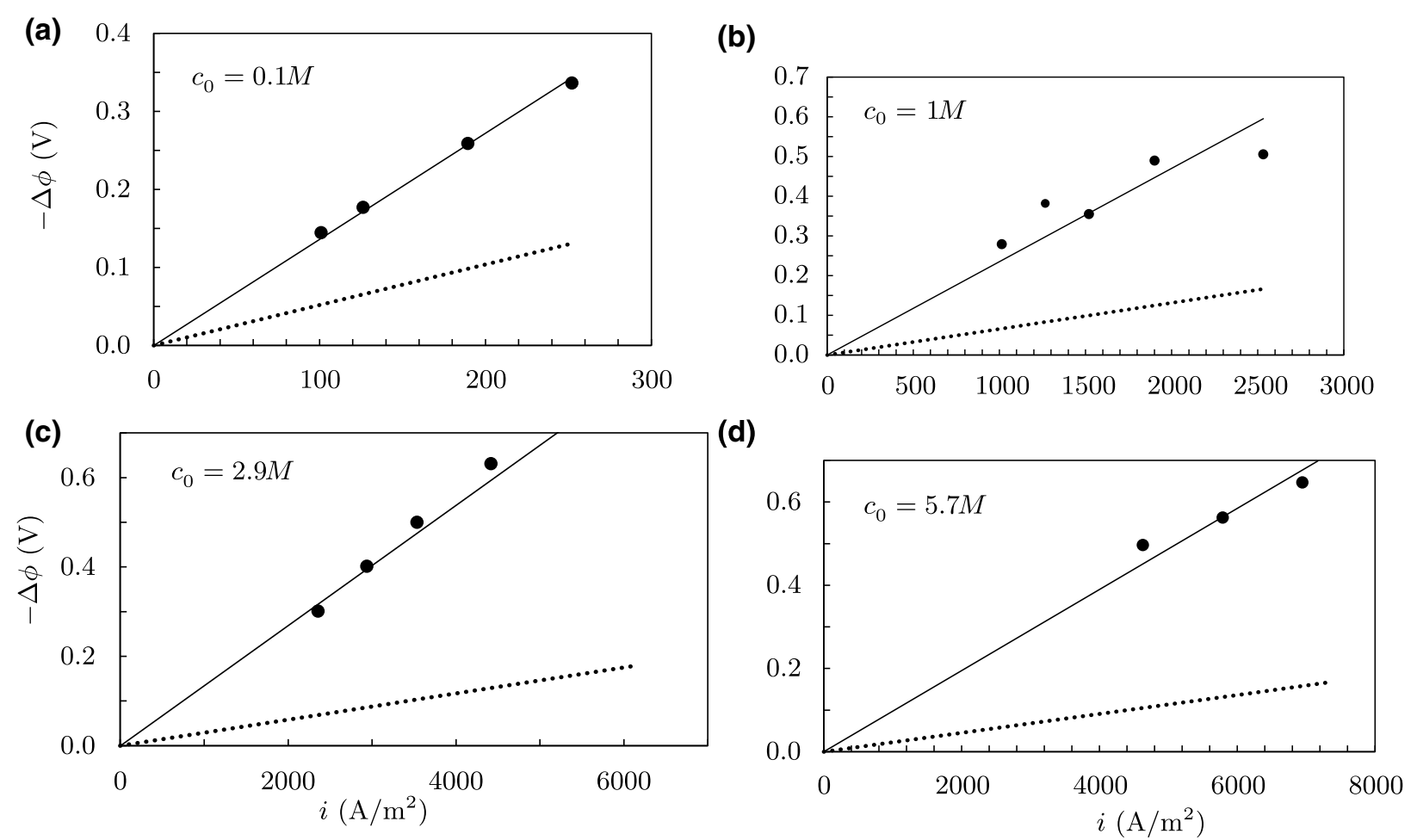

FIG. 13. The measured potential difference $-\Delta \phi_{\text {tot }}$ between the left and right bulk electrolytes $t=150 \mathrm{~s}$ after the current is switched on (data points), a linear fit (solid line), and the calculated $-\Delta \phi=i L / \kappa_{0}$ (dotted line).

threefold difference seems far too large to be explained by, for example, a larger tortuosity. Instead, it may be explained by the additional distance that the ionic current travels beyond the separator thickness $L$.

For simplicity, and in reasonable agreement with the data, we use a simple linear fit in Fig. 13.

To allow a comparison between the measured $\Delta \phi_{\text {tot }}$ and the theoretical $\Delta \phi$, we add to the latter ohmic drops $i l_{e} / \kappa_{l}$ and $i l_{e} / \kappa_{r}$, with $l_{e}$ obtained from the fitted slope in Fig. 13.
Note that here we use the same effective conductivity as in the separator, both for the anode and the cathode, depending only on the electrolyte concentration. The results given in Table I indicate that $l_{e}$ is a bit smaller than the electrode thickness for the lowest current density and roughly doubles for the higher current densities to a value slightly larger than the electrode thickness. The precise quantification and origin of this additional potential drop associated with the electrodes warrants further investigation.

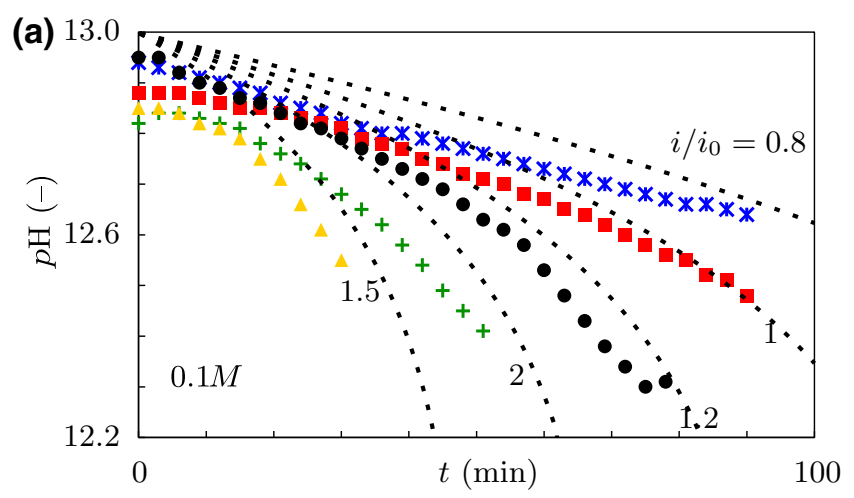

(b)

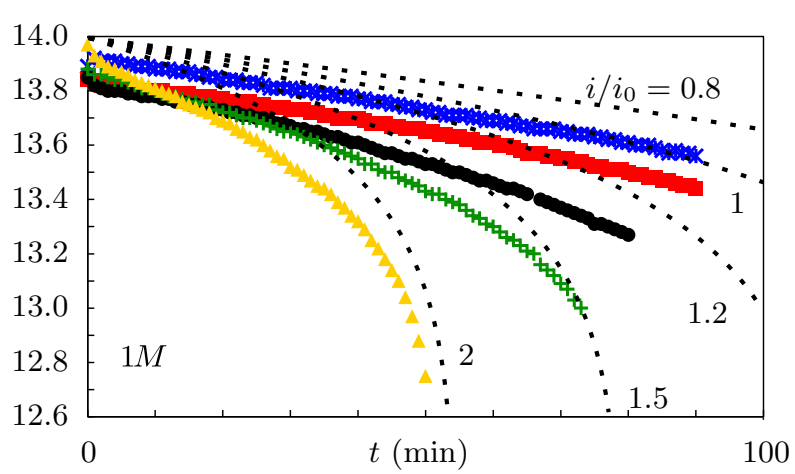

FIG. 14. The $p \mathrm{H}=14+\log _{10}\left(c_{l}[M]\right)$ for $c_{0}=0.1 M$ (a) and $c_{0}=1 M$ (b). As discussed below Eq. (F5), when $i \approx i_{0}$, the $p \mathrm{H}$ is expected to vary logarithmically initially for $t \ll t_{0}$. The dotted black lines are obtained by numerically solving Eqs. (28)-(32) and (22) using the values in Table II. The limited accuracy of the $p \mathrm{H}$ sensor, 0.1 unit of $p \mathrm{H}$, can be clearly seen from the values at $t=0$, which according to the molarity should be 13 and 14 at $0.1 M$ and $1 M$, respectively. 
3. Potential drop $\Delta \phi_{\text {tot }}$
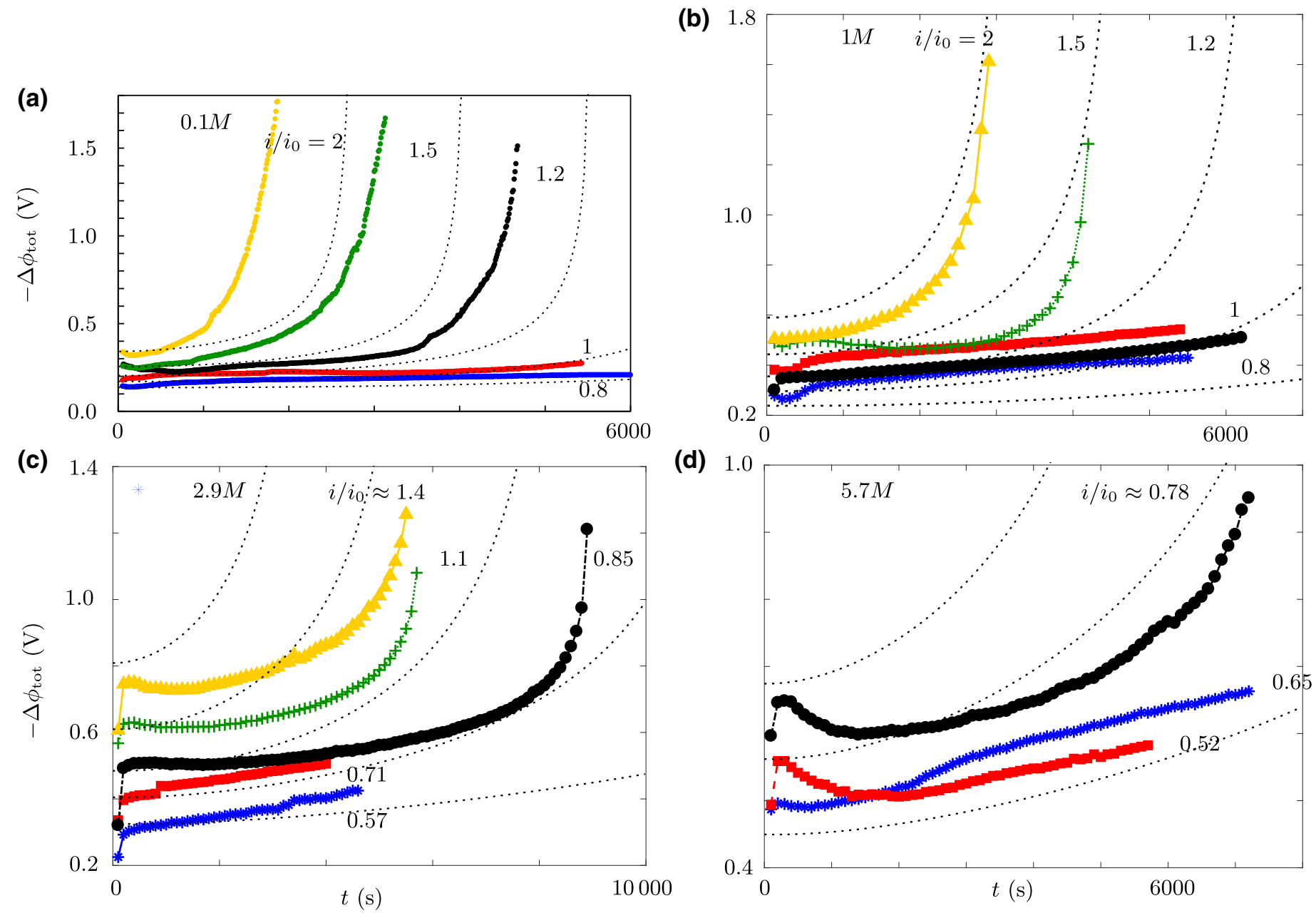

FIG. 15. The measured potential difference $-\Delta \phi_{\text {tot }}$ between the left and right electrolyte volumes, as a function of time $t$ after switching on different values of the current density $i / i_{0}$ and for various electrolyte concentrations. The dotted black lines give $-\Delta \phi+$ $i l_{e} / \kappa_{l}+i l_{e} / \kappa_{r}$, where $\Delta \phi, \kappa_{l}$, and $\kappa_{r}$ are obtained from Eqs. (28)-(32) and (22) and the values in Table II.

\section{B. Diffusional timescale}

In Fig. 15 we show a comparison between the measured potential $-\Delta \phi_{\text {tot }}$ and $-\Delta \phi+i l_{e} / \kappa_{l}+i l_{e} / \kappa_{r}$, obtained by numerically solving Eqs. (28)-(32) and (22), using the values in Tables I and II. Reasonable agreement is obtained for the time at which limiting conditions are approached. For the lowest concentration, this time is overestimated while, for the higher concentrations, it is somewhat underestimated. For $c_{0}=0.1 \mathrm{M}$, for example, experimental curves seem to be approximately $10 \mathrm{~min}$ ahead of the model curves in Fig. 15.

This may actually be due to our neglect of the time it takes to reach quasisteady concentration profiles inside the separator. As discussed in Ref. [35], there is a "lag time" in the flux of cations compared to that expected on the basis of a steady-state calculation, approximately given by $\left(L^{2} / 6 D_{a}\right)\left[\left(c_{r}-c_{l}\right) /\left(c_{r}-c_{l} e^{\mathrm{Pe}}\right)\right]$. This timescale diverges and becomes negative when $c_{r} / c_{l} \geq e^{\mathrm{Pe}}$. This is associated with a vanishing salt flux $N=0$ in Eq. (21), at which point the characteristic timescale for diffusion diverges and our assumption of a quasisteady state breaks down. As subtly argued in Ref. [35], a positive flow velocity adds a positive advective component to the flux $N$, but at the same time increases the oppositely directed diffusive flux by steepening the concentration profile near $x=L$ according to Eq. (19). As the diffusional time diverges over an extended period of time, the electrolyte flux continues even though $N=0$ according to steady state. As shown in Figs. 5 and 6 for $i / i_{0}=1.221$ and $\mathrm{Pe} \approx 0.7$, roughly between $t / \tau_{0}=0.4$ and 0.8 a steady-state calculation predicts the electrolyte flux becomes negative, while a transient calculation may reveal it to actually be positive. These rather special circumstances may be what causes the small sudden increases in the $c_{0}=0.1 M$ Fig. 15 for $t \approx 0.17 \tau_{0} \approx 1100 \mathrm{~s}, 0.4 \tau_{0} \approx 2600 \mathrm{~s}$, and $0.55 \tau_{0} \approx 3600 \mathrm{~s}$ for $i / i_{0}=2,1.5$, and 1.2, respectively. Around these times 
(a)

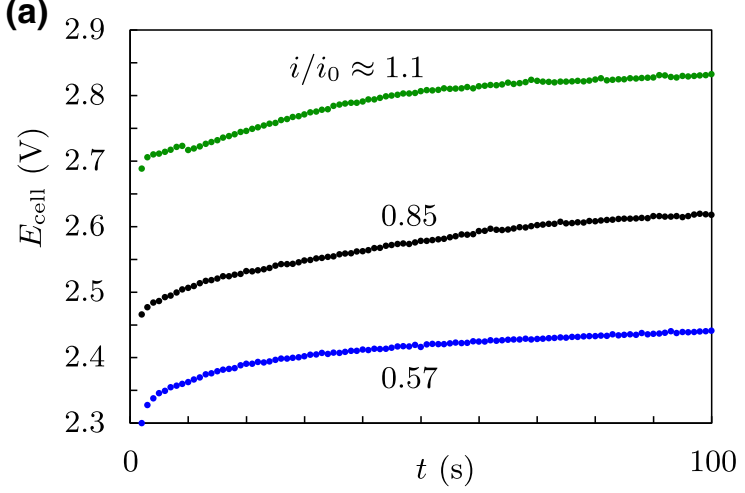

(c)

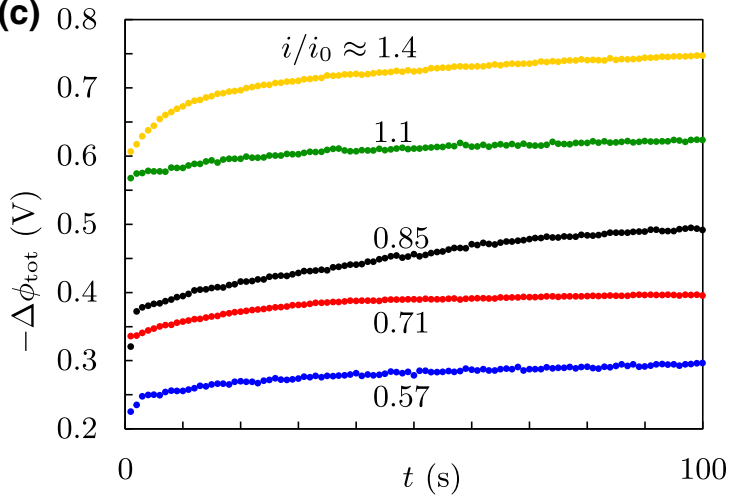

(b)

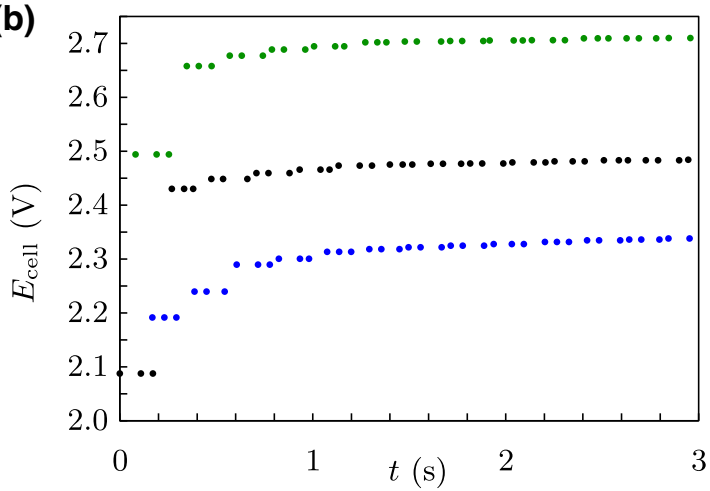

(d)

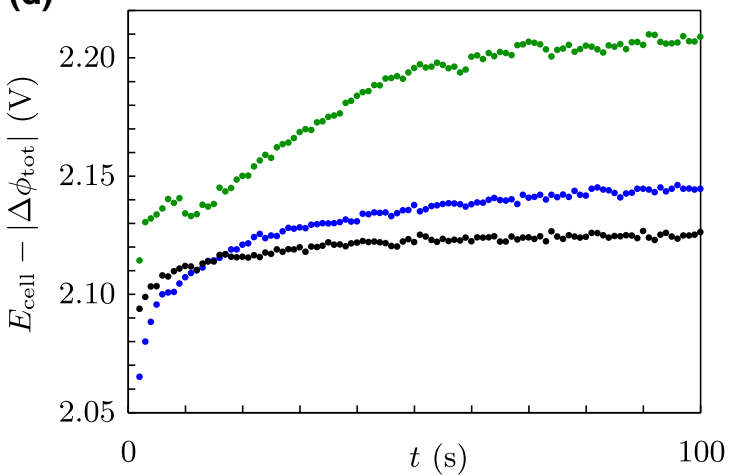

FIG. 16. The cell voltage $E_{\text {cell }}$ and the potential drop $-\Delta \phi_{\text {cell }}$ measured for an electrolyte concentration $c_{0}=2.9 M$ and various current densities $i / i_{0}$. Both go up with time. The cell voltage goes up more however so that the difference $E_{\text {cell }}-\left|\Delta \phi_{\text {cell }}\right|$ also increases with time.

a hiccup in the recorded levels can also be seen from Fig. 11. Here it would be interesting to extend the analysis of Ref. [35] to a nonconstant electro-osmotic flow velocity and also simulate the transient concentration profiles in the separator. Note from Table II that, for $c_{0}=1 M$ and $2.9 M$, the Péclet number is a bit lower, so these special conditions may not be met.

In Fig. 16 we show an increase in the recorded potential drop $\Delta \phi_{\text {tot }}$ and cell voltage $E_{\text {cell }}$ over the first $100 \mathrm{~s}$ after switching on the current for $c_{0}=2.9 \mathrm{M}$. This timescale is similar to $L^{2} / 4 D_{a}=\mathcal{O}\left(10^{2} \mathrm{~s}\right)$ [35], associated with diffusion in the separator. Note that various of the curves in Fig. 15 also show a decrease in $-\Delta \phi_{\text {tot }}$ before starting to increase as limiting conditions are approached. The decrease is much larger than that predicted by the diffusion potential, which is included in the simulations and shown in the same figure. These phenomena require further investigation to be explained with more certainty.

\section{Cell potential $\boldsymbol{E}_{\text {cell }}$}

In Fig. 16 we also show the cell voltage $E_{\text {cell }}$, the potential difference between the electrodes. In addition to $\Delta \phi$ and the open-circuit potential, this also includes the activation overpotentials required to drive the reaction, as illustrated by Eq. (33). These in turn are influenced by both bubbles and concentration effects. For very short timescales, there may also be a capacitive response due to charging of the electrical double layers in the system.

With $C$ and $a$ the capacity per unit surface area and the volumetric surface area, respectively, the total capacity per geometrical area of the separator is given by $a L C$. Multiplying with the areal separator resistance $L / \kappa$ gives a capacitive $R C$ time $a L^{2} C / \kappa$. With the rough numbers mentioned in Sec. II A and the conductivities from Table I, this gives a timescale of $\mathcal{O}(1 \mathrm{~s})$ for $c_{0}=1 M$ and $\mathcal{O}(10 \mathrm{~s})$ for $c_{0}=0.1 M$. In Figs. 16 and 17 we indeed show a rapid increase in the cell voltage over roughly these timescales.

As discussed in Sec. III C 2, when $i \approx i_{\text {lim }}$, the anolyte concentration initially decreases exponentially, in accordance with Eq. (29). The associated concentration overpotential in Eq. (33) is in this case $\Delta E_{\text {conc }} \approx b_{a} t / \tau$; see Eq. (F5). The slope of roughly $50 \mathrm{mV} / \mathrm{h}$ observed for $i / i_{0}=1$ in Fig. 17 would, with $\tau \approx 6.6 \times 10^{3} \mathrm{~s}$ from Table I, correspond to an anodic Tafel slope of $b_{a} \approx 0.1 \mathrm{~V}$. This is on the high side, but may correspond to a value doubled by ohmic limitations [38]. There is also however a rather large uncertainty in this data formed by subtracting two independently measured quantities. Note, for example, that, for $i / i_{0}=$ 0.8 , there seems to be no increase at all. For the higher 

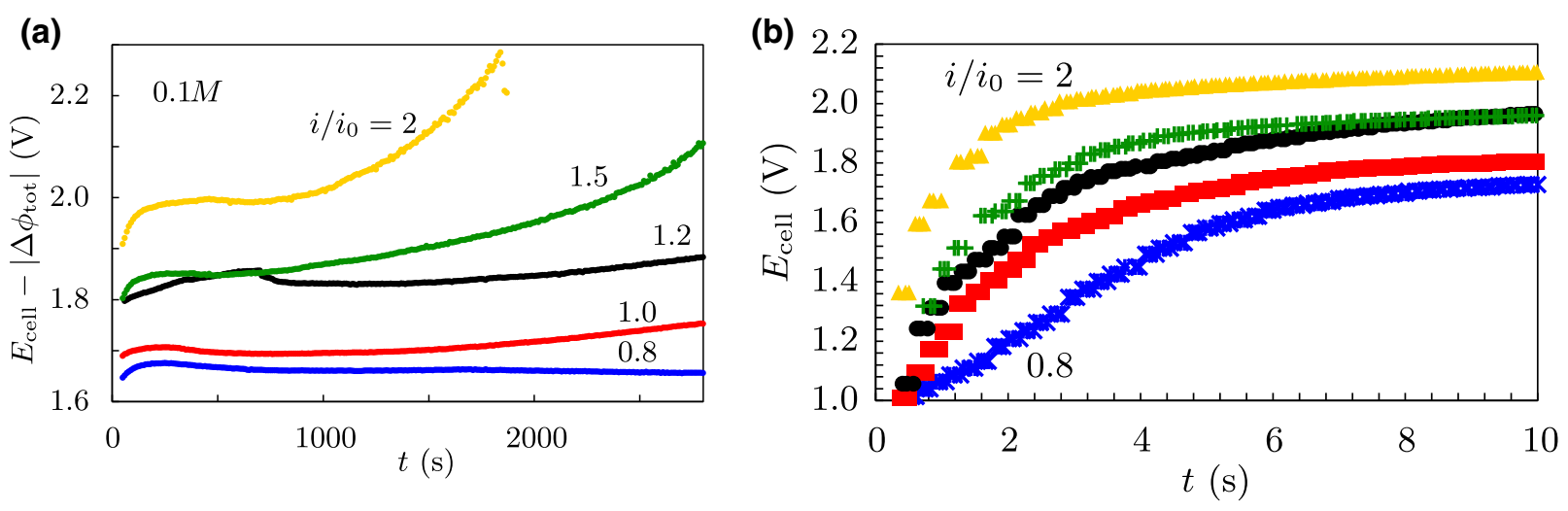

FIG. 17. The difference $E_{\text {cell }}-\left|\Delta \phi_{\text {tot }}\right|$, roughly equal to the activation overpotential (a), and the cell potential (b) for $c_{0}=0.1 M$. The increase in activation overpotential with time may be primarily attributed to an increase in the concentration overpotential. On a much faster timescale, possibly associated with capacitive effects, the cell potential can be seen to increase in the first seconds after the current is switched on. In Fig. 16 we show that, for a higher concentration, this timescale is even smaller.

values $i / i_{0}=1.5$ and 2 , a rather large increase can be seen associated with approaching the limiting conditions, in which case the concentration overpotential diverges.

\section{Electro-osmotic drag}

The level increase at the cathode and decrease at the anode is contrary to what one would expect from electro-osmotic drag of the reacting hydroxide anions, which move from cathode to anode. In the case of exchange membrane (PEM) water electrolysers, electroosmotic drag causes a net transport of water [42]. Protons drag along a solvation shell, or hydration shell, of water molecules, causing a net flow from anode to cathode. In alkaline water electrolysis, however, the primary current-carrying ion is the hydroxide ion, which moves in the opposite direction from cathode to anode.

Both hydroxide and potassium ions have a solvation number, or hydration number, of the order of $\lambda \approx 6 \pm 1$ $[43,44]$, so that each ion transports on average about six water molecules. The associated water flux will be given by $N_{w}^{\text {drag }} \approx \lambda\left(N_{+}+N_{-}\right)$or, using Eq. (15) with $\nu_{ \pm}=1$ and $z_{ \pm}= \pm 1$,

$$
N_{w}^{\text {drag }} \approx \lambda\left[2 N+\left(t_{+}-t_{-}\right) \frac{i}{F}\right] .
$$

To this we have to add the water flux due to electro-osmotic flow $N_{w}^{\text {flow }}=u c_{w}$, with $c_{w} \approx 55 M$ the molar concentration of water, to obtain the total water flux $N_{w}=N_{w}^{\text {drag }}+N_{w}^{\text {flow }}$.

We recall the modeled fluxes of Fig. 6, which may be used to illustrate how these vary over time throughout an experiment. At two instances the electrolyte flux $N$ in Fig. 6 vanishes, as advection to the right exactly cancels diffusion to the left, and Eq. (41) gives $N_{w}^{\text {drag }} \approx 3 i / F$. With $\Delta \phi \approx-i L / \kappa_{0}$, Eq. (6) gives the electro-osmotic flow water flux as $N_{w}^{\text {flow }} \approx \mathcal{M} c_{w} i / \kappa_{0}$. Using the data of Table I, this gives $N_{w}^{\text {flow }} \approx 50 i / F$ for $c_{0}=0.1 M$, so that in this case the electro-osmotic drag can be neglected. On the other hand, for $c_{0}=5.7 \mathrm{M}$, we find that $N_{w}^{\text {flow }} \approx 0.7 i / F$, indicating that electro-osmotic drag should dominate over electro-osmotic flow.

The mobility $\mathcal{M}$ from Table I used in the estimate of $N_{w}^{\text {flow }}$ is however directly derived from the net water flux $N_{w}=N_{w}^{\text {flow }}+N_{w}^{\text {drag }}$ as obtained from the observed electrolyte levels and thus already effectively includes the effect of electro-osmotic drag. Despite the net electroosmotic drag of water to the left, the net water flux is still observed to be to the right. This implies that the actual electro-osmotic flow mobilities $\mathcal{M}^{\text {flow }}=u /(-\Delta \phi / L)$ are substantially higher than the reported mobilities $\mathcal{M}=$ $\left(N_{w} / c_{w}\right) /(-\Delta \phi / L)$, which use the superficial water flux $N / c_{w}$ rather than $u$. Neglecting concentration gradients we can use $N=u c_{0}$ and Eq. (25) to write Eq. (41), and using Eqs. (18) and (11) with $v_{ \pm}=1$ and $z_{ \pm}= \pm 1$, we can write $\mathcal{M}=\mathcal{M}^{\text {flow }}+\mathcal{M}^{\text {drag }}$ as

$$
\mathcal{M} \approx \mathcal{M}^{\text {flow }}\left(1+2 \lambda \frac{c_{0}}{c_{w}}\right)+\lambda\left(D_{+}-D_{-}\right) \frac{F}{R T} \frac{c_{0}}{c_{w}} .
$$

Here the terms proportional to $\lambda$ represent the electroosmotic drag contribution from Eq. (41). The combined mobility $\mathcal{M}$ obtained from the recorded liquid levels is given in Table I. We also give the electro-osmotic flow mobility $\mathcal{M}^{\text {flow }}$ as calculated from Eq. (42). For $c_{0}=$ $5.7 M$, this mobility is a factor $\mathcal{M}^{\text {flow }} / \mathcal{M} \approx 5$ higher. This shows that the net ionic drag to the left cancels to a large degree the electro-osmotic flow that would otherwise be present. As a result, the effective zeta potential shown in Ref. [31] likely strongly underestimates the actual zeta potential at these higher concentrations. Note that, contrary to $\mathcal{M}$, the calculated electro-osmotic flow mobility $\mathcal{M}^{\text {flow }}$ increases with increasing concentration. 
In general, the net superficial volumetric water flux $N_{w} / c_{w}$ can differ from the flow velocity $u$ and may be modeled as such. At elevated concentrations however it becomes impossible to distinguish between these two quantities. For $c_{0}=5.7 M$, for example, the concentration of water molecules $2 \lambda c_{0}$ in the solvation shells of the electrolyte ions would even exceed the amount of water molecules $c_{w}$ present, leaving no water molecules for any flow. Insight from molecular dynamics simulations, can perhaps in the future provide guidance on how to more accurately take these effects into consideration. For now, however, we take $u$ to be equal to $N_{w} / c_{w}$ so that we can derive $\mathcal{M}$ directly from the liquid levels. Despite a negative flux $N_{w}^{\mathrm{drag}} \approx \lambda\left(N_{+}+N_{-}\right)<0$, caused by ions dragging water to the left, the net water transport is to the right. The electric force on a thin positively charged layer near the surface is thus effective in creating a net electro-osmotic flow opposing the ionic drag.

Although this may be reading too much into the data, given its experimental inaccuracies, in Fig. 11 for $c_{0}=$ $5.7 M$ and $i / i_{0}=0.52$ and 0.65 , the levels initially increase at about twice the rate of that after half an hour. As illustrated in Fig. 6, the electro-osmotic drag $N_{w}^{\text {drag }} \approx \lambda\left(N_{+}+\right.$ $N_{-}$) becomes more negative as time proceeds. This is due to a decrease in salt flux $N$ associated with an increasing diffusive flux to the left opposing the advective flux to the right. This results in an enhanced drag of solvated water molecules to the left, diminishing the net rate of level difference. An alternative explanation, however, is that the hydrostatic pressure caused by the increasing liquid level on the right opposes the flow to the right. Eventually, the net water flux can also be seen to increase again. This may be explained by an increase in electro-osmotic flow, driven by the increasing potential difference.

Note that the solvation shell of ions does not necessarily contain all species in proportion to their abundance in the electrolyte, but actually typically under represents dissolved gases. In this case the advective transport of gases like oxygen and hydrogen will be higher than predicted on the basis of the measured effective electro-osmotic velocity $u$, which at high electrolyte concentrations will in large part be due to water drag. Note also that at still higher concentrations or slightly lower electro-osmotic flow mobilities $\mathcal{M}^{\text {flow }}$, electro-osmotic drag may dominate over electroosmotic flow. This would give rise to a flow reversal, but without an accompanying charge inversion $[17,45]$.

\section{E. Further discussion}

Besides significant uncertainty in the various model input parameters, there are also further errors associated with our assumptions in the model. A known modeling error is associated with our use of dilute solution theory. To partially take concentrated-solution effects into account, instead of Eqs. (13) and (18) for the ambipolar diffusivity
$D_{a}$ and conductivity $\kappa$, we use the empirical values listed in Table I. We take these properties to be constant in the modeling and simulations, but these will depend on, for example, concentration and temperature, which both vary throughout the experiment.

The electrolyte conductivity, for example, in the dilute approximation varies linearly with electrolyte concentration. For various electrolytes, this approximately holds up to concentrations of the order of one or even several molar, before leveling off to a maximum, beyond which the conductivity decreases again. For $\mathrm{KOH}$ at room temperature, the maximum conductivity occurs close the $5.7 M$ we use in our experiments. Since the used dilute limit equations assume a linear dependence on concentration, when the concentration increases locally, the conductivity is erroneously predicted to increase, while the predicted decrease with decreasing local concentration is overestimated.

As discussed in Sec. IV D, the water flux through the separator $N_{w}$ is partly driven by ion fluxes, which are throughout an experiment not always strictly proportional to the potential drop $\Delta \phi$. Therefore, the mobility $\mathcal{M}=$ $\left(N_{w} / c_{w}\right) /(-\Delta \phi / L)$ will vary throughout an experiment, introducing another known error into our simple modeling in which we use a constant value. We also note that the $0.1 M$ and $1 M$ results of Fig. 12 are described slightly better with an offset-linear relationship in which a finite voltage of $\Delta \phi \approx 35 \mathrm{mV}$ is required to initiate flow. Since there does not seem to be any theoretical basis for this, we attribute this to measurement inaccuracies and use a linear relationship.

The permeability of the porous separator used is $k \approx$ $7 \times 10^{-16} \mathrm{~m}^{2}$ [46] and the electrolyte viscosity is in the range $\mu \sim 1-2 \mathrm{mPa}$. The observed flow velocities of the order of $u \sim 1 \mu \mathrm{m} / \mathrm{s}$ can then also be reached with a pressure difference of approximately $1 \mathrm{kPa}$. This corresponds to a hydrostatic pressure of only approximately $0.1 \mathrm{~m}$ of liquid. Such level differences do actually arise over time in our experiments, something that can be easily taken into account in a model by including $\Delta p$ in the derivation of Eq. (32). The nonconstant flow profile associated with superimposed electro-osmotic and pressure flows can lead to Taylor dispersion [47]. Because the Péclet number using the pore radius as a length scale is however very small, this effect, included in, e.g., Ref. [48], can safely be neglected compared to molecular diffusion.

As discussed in Ref. [31], flows through the separator can contribute significantly to undesirable transport of dissolved gases. In most electrolysers the pressure between anolyte and catholyte are balanced, but our findings here shows that controlling the liquid levels can also be used as a means to counteract electro-osmotic flows through the separator. At $5.7 M$ the limiting current is $7 \mathrm{kA} / \mathrm{m}^{2}$, in the range of operating conditions of modern alkaline electrolysers. Nonetheless, in practice a limiting current will rarely 
be observed because in most electrolysers the anolyte and catholyte are mixed. An open connection between anolyte and catholyte provides a convenient way to equilibrate pressure, $\mathrm{KOH}$ concentration, and simplify water management. To minimize the resulting mixing of dissolved hydrogen and oxygen [49], a recombination of electrolyte streams may also be done periodically [50] similar to in PEM electrolysers [42].

\section{CONCLUSIONS}

Taking into account diffusion, migration, and advection of an electrically neutral binary electrolyte with arbitrary valencies, we derive a useful analytical expression, Eq. (24), for the potential drop over a neutral porous layer. Considering a timescale much larger than the diffusional timescale, we derive Eq. (29), describing the time evolution of the concentrations on either side of the layer. We consider various limits, analytical solutions, extensions, and comparisons with previous results from different disciplines.

We experimentally validate the derived equations by investigating the transport through a microporous separator used in alkaline water electrolysis. Electro-osmotic flows from anode to cathode are found to develop as a result of the potential drop over the separator, which somewhat lower the limiting current density. While such limitations are in practice avoided by recombining anolyte and catholyte, this can lead to undesirable cross over of dissolved gases. Similarly, the flows themselves can significantly impact dissolved gases. Estimation of the detailed impact is complicated by opposing electro-osmotic drag effects, discussed in Sec. IV D.

The timescales associated with draining the electrolyte volume and reaching a quasisteady state or limiting current condition are both included in the model equations. From the experiments we observe two more distinct timescales. On the order of a second, the cell voltage is found to increase rapidly, plausibly associated with the capacitive charging of the separator double layers. On the order of a minute, both the ohmic and activation overpotentials are found to increase further, which will require further investigation to explain with certainty.

Even though the electrodes are positioned in a zerogap configuration, a substantial ohmic drop is measured associated with a distance of the order of the electrode thickness.

We thus see that the effective combination of theory and experiments used in this work leads to reasonable agreement but also several instances where additional phenomena turn out to play a role. The generality and relative simplicity of the used theory goes a long way to insightfully describe the main physics, while the experiments point at a rich variety of additional phenomena.

\section{ACKNOWLEDGMENTS}

I gratefully acknowledge H. Rajaei, T.P.M. Hooijschuur, T.M. van Velzen and M.M. het Lam for their help with the experiments and R. Hartkamp for insightful discussions.

\section{APPENDIX A: ALTERNATIVE EXPRESSIONS FOR $\Delta \phi$}

\section{As a function of $N$}

Considering the limiting conditions associated with $c_{l}=$ 0, we can use Eq. (21) to write

$$
\frac{c_{l}}{c_{r}}=e^{-\mathrm{Pe}}\left(1-\frac{N}{N_{\mathrm{lim}}}\right),
$$

where

$$
\begin{aligned}
N_{\lim } & =\frac{u c_{r}}{1-e^{\mathrm{Pe}}} \\
& =-\frac{D_{a} c_{r}}{L} \frac{\mathrm{Pe}}{e^{\mathrm{Pe}}-1} \approx \begin{cases}-u c_{r} / e^{\mathrm{Pe}}, & \mathrm{Pe} \gg 1, \\
-D_{a} c_{r} / L, & |\mathrm{Pe}| \ll 1, \\
u c_{r}, & \mathrm{Pe} \ll-1 .\end{cases}
\end{aligned}
$$

We see that in the case of a flow to the right, there is a maximum flux $-N$ that can be sustained towards the left by diffusion and advection, without $c_{l}$ vanishing. The larger the velocity, the smaller this flux. See Fig. 2 for an illustration. Inserting Eq. (A1) into Eq. (22) or (24) gives the potential drop in terms of $i$ and $N$ as

$$
\Delta \phi=\left(\chi-\frac{i L}{\kappa_{\mathrm{c}}} \frac{1}{\mathrm{Pe}}\right) \ln \left(1-\frac{N}{N_{\mathrm{lim}}}\right)-\chi \mathrm{Pe},
$$

where

$$
\frac{i L}{\kappa_{\mathrm{c}}} \frac{1}{\mathrm{Pe}}=\frac{i D_{a}}{N} \frac{c}{\kappa}=t_{+} t_{-} \frac{1 / z_{+}-1 / z_{-}}{z_{+} \nu_{+}} \frac{R T}{F} \frac{i / F}{N} .
$$

In an ideal separator or perfect filtration membrane, no salt goes through so that $N=0$. In this limit Eq. (A3) gives, with $\kappa_{c}=(N / u c) \kappa$,

$$
\Delta \phi=-\frac{i L}{\kappa_{r}} \frac{e^{\mathrm{Pe}}-1}{\mathrm{Pe}}-\chi \mathrm{Pe} \quad(N=0) .
$$

The final term $-\chi \mathrm{Pe}$ is the diffusion potential due to the concentration difference $c_{r} / c_{l}=e^{\mathrm{Pe}}$ that has to be present to maintain $N=0$ in Eq. (21). This concentration difference induced by convective flow is routinely measured in steady-state convective diffusion or convective electrophoresis experiments at zero current and salt flux to determine the thickness-to-area ratio of (biological) membranes $[2,3]$.

When we want to consider the limiting conditions associated with $c_{r}=0$, instead of Eq. (A1), 
we can write $c_{r} / c_{l}=e^{\mathrm{Pe}}\left(1-N / N_{\lim }^{r}\right)$ with $N_{\lim }^{r}=c_{l} u /$ $\left(1-e^{-\mathrm{Pe}}\right)=-\left(D_{a} c_{l} / L\right)\left[-\mathrm{Pe} /\left(e^{-\mathrm{Pe}}-1\right)\right]$. Equivalently, we can change the direction of the $x$ axis, reversing $l$ and $r$ and changing the signs of $i, \mathrm{Pe}$, and $\Delta \phi$.

\section{As a function of $N_{i}$}

The average electric field in the $x$ direction, $-\Delta \phi / L$, can immediately be obtained from Eq. (24) or (A3). The local electric field is given by Eq. (16) as $-\phi^{\prime}=i / \kappa+\chi\left(c^{\prime} / c\right)$. Using Eq. (16) and Eq. (19), this can be written explicitly as a function of $x$ as

$$
\begin{aligned}
-\phi^{\prime}= & \frac{i}{\kappa_{l}+\left(\kappa_{r}-\kappa_{l}\right)\left(e^{\mathrm{Pe}(x / L)}-1\right) /\left(e^{\mathrm{Pe}}-1\right)} \\
& +\frac{\chi}{L} \frac{\mathrm{Pe}}{1+\left(c_{l} e^{\mathrm{Pe}[(L-x) / L]}-c_{r} e^{-\operatorname{Pe}(x / L)}\right) /\left(c_{r}-c_{l}\right)} .
\end{aligned}
$$

The second diffusional contribution to the electric field opposes the first term when $\chi\left(c_{r}-c_{l}\right) / i<0$ and adds to it when $\chi\left(c_{r}-c_{l}\right) / i>0$. Equation (15), inserting Eq. (21) for $N$ and Eq. (24) for $i$, gives the species flux $N_{i}$ in terms of only concentration and potential differences as

$$
\begin{aligned}
N_{i}= & u v_{i} \frac{c_{l} e^{\mathrm{Pe}}-c_{r}}{e^{\mathrm{Pe}}-1}+\frac{t_{i}}{F z_{i}} \frac{\kappa_{\mathrm{c}}}{L} \frac{\Delta \phi+\chi \ln \left(c_{r} / c_{l}\right)}{\ln \left(c_{r} / c_{l}\right) / \mathrm{Pe}-1} \\
= & u v_{i} \frac{c_{l} e^{\mathrm{Pe}}-c_{r}}{e^{\mathrm{Pe}}-1} \frac{D_{i}}{D_{a}} \frac{\ln \left(c_{r} / c_{l}\right)-D_{a} \mathrm{Pe} / D_{i}+z_{i} F \Delta \phi / R T}{\ln \left(c_{r} / c_{l}\right)-\mathrm{Pe}} .
\end{aligned}
$$

Here we used Eq. (18) to write $\left(t_{i} / F z_{i} v_{i} D_{a}\right)(\kappa / c)=$ $\left(z_{i} D_{i} / D_{a}\right)(F / R T)$ and we used $1+\left(z_{i} D_{i} / D_{a}\right)(F \chi / R T)=$ $D_{i} / D_{a}$. So far, we have provided expressions for $\Delta \phi$ in terms of $i, c_{l}$, and $c_{r}$ in Eq. (24), or $i$ and $N$ in Eq. (A3). Equation (A6) readily gives $\Delta \phi$ in terms of $N_{i}$ and the concentrations $c_{l}$ and $c_{r}$. We can also use Eq. (15) in Eq. (A3) to write $\Delta \phi$ in terms of $N_{i}$ and $N$ or $N_{i}$ and $i$.

\section{APPENDIX B: A NONMOBILE ION $\left(N_{n}=0\right)$}

\section{General}

Near ion-exchange membranes, or in the case of a steady-state reaction involving only one ion, the flux of a "nonmobile" ion with index $i=n$ vanishes, so that $N_{n}=0$ and Eq. (15) gives

$$
N=-\frac{t_{n}}{F z_{n} v_{n}} i
$$

Therefore, we can write $i / i_{\text {lim }}$ instead of $N / N_{\text {lim }}$, with $i_{\text {lim }}=-\left(F z_{n} v_{n} / t_{n}\right) N_{\text {lim }}$. With Eqs. (A2), this gives

$$
i_{\lim }=-\frac{D_{a}}{t_{n}} \frac{F z_{m} v_{m} c_{r}}{L} \frac{\mathrm{Pe}}{e^{\mathrm{Pe}}-1} .
$$

Denoting the index $i$ of the mobile ion with $m$, with Eqs. (13) and (11) we have

$$
\frac{D_{a}}{t_{n}}=\left(1+\left|\frac{z_{m}}{z_{n}}\right|\right) D_{m} .
$$

The limiting current is proportional to the diffusivity of the mobile ion, as expected. The additional factor $1+\left|z_{m} / z_{n}\right|$ accounts for the additional current due to migration and is the only quantity in which the nonmobile ion plays a role. For a monovalent electrolyte, $1+\left|z_{m} / z_{n}\right|=2$ so that, due to migration, the limiting current is twice the diffusional current. The numerator of the advection correction $\mathrm{Pe} /\left(e^{\mathrm{Pe}}-1\right)$ of Eq. (A2) reflects the convective flux contribution, while the denominator takes into account the effect flow has on the diffusive flux through the steepening of the concentration profile.

From Eq. (B2), the condition $i / i_{\lim }>0$ requires $i / z_{m}<$ 0 , so that the flux of mobile charge should be to the left. In this case a limiting current associated with $c_{l}=0$ can be obtained. This is due to our choice of defining $N_{\mathrm{lim}}$ as in Eq. (A1). If $i / i_{\lim }<0$, a limiting current exists associated with $c_{r}=0$.

For a flow to the right, $\mathrm{Pe}>0$, and the advective flux opposes the diffusional flux of species to the left. Therefore, there is a positive limiting current associated with $c_{l}=0$, carried by anions $\left(N_{+}=0\right)$, and a negative limiting current carried by cations $\left(N_{-}=0\right)$. Flow in this case reduces the magnitude of the limiting current as $\mathrm{Pe} /\left(e^{\mathrm{Pe}}-1\right)<1$. See Fig. 2 for an illustration of how flow impacts the concentration profile and associated diffusive flux and limiting current.

For a flow to the left, $\mathrm{Pe}<0$, and the advective flux opposes the diffusional flux of species to the right. Therefore, there is a negative limiting current associated with $c_{l}=0$, carried by anions $\left(N_{+}=0\right)$, and a positive limiting current carried by cations $\left(N_{-}=0\right)$. See Fig. 2 for an illustration of how flow impacts the concentration profile and associated diffusive flux and limiting current.

It is important to note that a limiting current does not exist when the constraint $N_{n}=0$ is dropped, as in the more general Eq. (22). Without this constraint, a current can be sustained solely by migration without any concentration differences, and hence without any diffusion limitations.

\section{Without flow}

In the limit $\mathrm{Pe} \rightarrow 0$ Eq. (B2) gives $i_{\mathrm{lim}}=z_{n} F\left(D_{a} / t_{n}\right)$ $\left(v_{n} c_{r} / L\right)$. From Eqs. (B1), (21), and (A2) we have $i=$ $i_{\lim }\left[\left(c_{r}-c_{l}\right) / c_{r}\right]$, so Eq. (26) can be written as $\Delta \phi=$ $\left(\chi+i_{\lim } L / \kappa_{r}\right) \ln \left(1-i / i_{\lim }\right)$. From Eqs. (18), (8), and (11) we find that $i_{\lim } L / \kappa_{r}=\left(D_{a} / z_{n} D_{n}\right)(R T / F)$, so with some algebra we can write $\chi-i_{\lim } L / \kappa_{r}=R T / F z_{n}$ and

$$
\Delta \phi=\frac{R T}{F z_{n}} \ln \left(1-\frac{i}{i_{\lim }}\right) .
$$


This simple result could have of course been obtained in a much simpler way from Eq. (10). With $N_{n}=0$ we see that the concentration satisfies a Boltzmann distribution so that $\ln c_{l} / c_{r}=z_{n} F \Delta \phi / R T$, giving Eq. (B4). Solving Eq. (B4) for $i$ gives

$$
i=i_{\lim }\left(1-e^{F z_{n} \Delta \phi / R T}\right) .
$$

These equations show a clear asymmetry in the direction of the current on the potential drop $\Delta \phi$, called ionic current rectification. As remarked in, e.g., Ref. [19], this currentvoltage relation is equivalent to that of an ideal (Schottky) diode. In this case $i_{\text {lim }}$ is referred to as the reverse saturation current.

An integral conductance of the layer $\kappa_{\text {eff }} \equiv-L(d i / d \Delta \phi)$ can be defined as the derivative of the current density with respect to the average electric field $-\Delta \phi / L$. From Eq. (B4) or (B5), assuming constant $c_{r}$ so that $i_{\text {lim }}$ is constant, we obtain $\kappa_{\text {eff }}=\left(F z_{n} / R T\right)\left(i_{\lim }-i\right) L$, or, with Eq. (8),

$$
\kappa_{\mathrm{eff}}=\left(1+\left|\frac{z_{m}}{z_{n}}\right|\right) \frac{\left|z_{m} z_{n}\right| F^{2} D_{m}}{R T} v_{m} c_{r}\left(1-\frac{i}{i_{\mathrm{lim}}}\right),
$$

proportional to the diffusivity and concentration of the mobile ion as well as the mixed $z_{m} z_{n}$ and the migrational multiplier $1+\left|z_{m} / z_{n}\right|$. As the limiting current density is approached, the effective conductivity drops to zero, as ever larger potential drops are required to maintain a zero flux of the nonmobile species.

With $N_{\text {lim }}=-D_{a} c_{r} / L$ from Eq. (A2) and defining $N_{L} \equiv-\left[D_{a}\left(c_{l}+c_{r}\right)\right] / L$, we can write Eq. (21) as $N=D_{a}\left[\left(c_{l}-c_{r}\right) / L\right]=2 N_{\lim }-N_{L}$ so that $1-N / N_{\lim }=$ $\left(1-N / N_{L}\right) /\left(1+N / N_{L}\right)$. With Eq. (B1) we can write $N / N_{L}=i / i_{L}$ with the limiting current density $i_{L}=$ $z_{n} F\left(D_{a} / t_{n}\right)\left\{\left[v_{n}\left(c_{l}+c_{r}\right)\right] / L\right\}$ sometimes referred to as Peers equation [51,52]. Using this in Eq. (26), with some algebra, we obtain the compact result often found in the literature [53-55],

$$
\Delta \phi=\frac{R T}{F z_{n}} \ln \left(\frac{1-i / i_{L}}{1+i / i_{L}}\right)
$$

or

$$
i=i_{L} \frac{1-e^{F z_{n} \Delta \phi / R T}}{1+e^{F z_{n} \Delta \phi / R T}}
$$

These equations are fully equivalent to Eqs. (B4) and (B5), respectively. The latter equations will however typically be more useful when $c_{r}$ is fixed so that $i_{\lim }$ is constant, while Eqs. (B7) and (B8) will be more useful when $c_{l}+c_{r}$ and, therefore, $i_{L}$ are constant.
Assuming constant $i_{L}$, the effective conductivity $\kappa_{\text {eff }} \equiv$ $-L(d i / d \Delta \phi)=L i_{L}\left(F z_{n} / R T\right)\left[\left(1-i^{2} / i_{L}^{2}\right) / 2\right]$ can be written as

$$
\kappa_{\mathrm{eff}}=\left(1+\left|\frac{z_{m}}{z_{n}}\right|\right) \frac{\left|z_{m} z_{n}\right| F^{2} D_{m}}{R T} \frac{v_{m}\left(c_{l}+c_{r}\right)}{2}\left(1-\frac{i^{2}}{i_{L}^{2}}\right),
$$

which is similar to Eq. (B6) apart from the quadratic rather than linear dependence on $i / i_{L}$.

\section{APPENDIX C: COMPARISON WITH PREVIOUS WORK}

\section{Ion concentration polarization}

Equation (B2) for the limiting current including the effect of advection was derived in Ref. [6] for a monovalent electrolyte with $z_{+}=-z_{-}=1$. Seemingly unaware of this earlier work, Nikonenko et al. [56] rederived these results using the same a priori unknown "effective" or "integral transference numbers" used in Ref. [6], which are different from our definition in Eq. (11). Both works however did not provide expressions for the potential drop $\Delta \phi$. Zholkovskii [5] did derive this, and combined several layers in the description of an electrodialysis cell, but only for equal anion and cation diffusion coefficients.

More recently, Khair [15] used the result of Tanaka [6] to derive the second term of Eq. (24) for a symmetric $(\chi=0)$ electrolyte in a neutral cation-exchange membrane and also matched these results to an analysis of the double layer, similar to Fleury et al. [11].

The combination of advection, diffusion, and migration of binary electrolytes has also been analyzed in the context of ramified electrodeposition in which the layer growth induces a liquid velocity $[11,12]$ as well as in the context of ion-selective membranes $[6,14,15]$.

\section{Filtration}

In the limit of zero current there have been earlier derivations of the "electric filtration potential" of Eq. (22) in the context of reverse osmosis or nanofiltration. These analyses [7,57] also include ion-specific reflection coefficients [58] in the advection terms of Eq. (10). These analyses typically also include a nonzero membrane charge $[2,3,59,60]$ that cannot be easily set to zero. Yaroshchuk [16] for example also considered electroneutral regions, but again did not explicitly calculate the potential drop. The more recent Ref. [61] gives in equation 27 the first two terms of Eq. (24) but provides the final logarithmic term as an unevaluated integral to allow for concentrationdependencies of the transport properties.

We can easily adjust the above equations to allow for nonunity "convective hindrance factors" or "ion transmission coefficients" $\tau_{i}$ that multiply the advective fluxes in 
Eq. (10) to give $\tau_{i} u c_{i}$. The resulting equation is sometimes referred to as the extended Nernst-Planck equation. A value of $\tau_{i}$ below 1 can in the case of large molecules be due to steric hindering, but for ions, is typically due to electrostatic interactions with the membrane. From the definition of $N$, introducing these factors $\tau_{i}$, has as a consequence that in Eq. (12), and all the equations below it, the superficial velocity $u$, and thus also Pe, are multiplied with the "salt transmission coefficient" $T=t_{-} \tau_{+}+t_{+} \tau_{-}$.

For $u>0$, the "effluent condition" $N_{i}=u c_{i, r}$ gives, using Eq. (15) with $i=0, N=u c_{r}$ also. Using this in Eq. (21), with the discussed modification $\mathrm{Pe} \rightarrow T \mathrm{Pe}$, immediately gives the ion retention or ion rejection coefficient $r \equiv 1-c_{r} / c_{l}$ as

$$
r=R \frac{e^{T \mathrm{Pe}}-1}{e^{T \mathrm{Pe}}-R}
$$

analogous to the widely applied Spiegler-Kedem expression derived for neutral species [62]. The ion rejection varies monotonically between $r \approx R \mathrm{Pe}$, when $T \mathrm{Pe} \ll$ 1 , and the "salt reflection coefficient" $R \equiv 1-T$, when $T$ Pe $\gg 1$. The assumption of constant transport coefficient values is a good approximation in the dilute limit, but for large electrolyte concentrations, variations should be taken into account to provide accurate predictions. A more formal analysis in terms of membrane sections and virtual solutions gives a similar system of equations, but allows for concentration dependencies of the effective diffusivities, confusingly referred to as permeabilities in this case.

We can now also calculate the effect of ion selectivity on the potential drop $\Delta \phi$. The introduction of $\tau_{i}$ leaves Eq. (15) unchanged, but adds to Eq. (14) an additional "streaming current" $i_{s}=\rho_{s} u$, where, with Eq. (8), $\rho_{s}=F z_{+} \nu_{+}\left(\tau_{+}-\tau_{-}\right) c$ is the average electrokinetic charge density. See, for example, Ref. [63] for a simple analysis giving the streaming current density as $\rho_{s}=$ $-\Delta p \varepsilon_{r} \varepsilon_{0} \zeta / \mu u=12 \epsilon \varepsilon_{0} \zeta / h^{2}$ for a planar channel of height $h$ with zeta potential $\zeta$. Integrating Eq. (16) generalizes Eq. (24) to

$$
\begin{aligned}
\Delta \phi & =-\frac{i L}{\kappa_{\mathrm{c}}}\left(1-\frac{\ln \left(c_{r} / c_{l}\right)}{T \mathrm{Pe}}\right) \\
- & \frac{R T}{F} \frac{\left(D_{+}-D_{-}\right) \ln \left(c_{r} / c_{l}\right)+\left(\tau_{+}-\tau_{-}\right) u L}{z_{+} D_{+}-z_{-} D_{-}},
\end{aligned}
$$

where the additional final term is the drop in streaming potential $-\int\left(\rho_{s} u / \kappa\right) d x$. In filtration applications usually $i=0$, reducing this expression to that of Ref. [7]. In the limit $T \mathrm{Pe} \ll 1$, Eq. (C1) gives $\ln \left(c_{r} / c_{l}\right)=\ln (1-r) \approx$ $-r \approx-R \mathrm{Pe}$ so that, for $i=0$, Eq. (C2) becomes

$$
\Delta \phi \approx \frac{R T}{F} \frac{\left(D_{+}-D_{-}\right)(1-T)-\left(\tau_{+}-\tau_{-}\right) D_{a}}{z_{+} D_{+}-z_{-} D_{-}} \mathrm{Pe} .
$$

As remarked in Ref. [7], this filtration potential, generated as a response to the flow, can be both positive and negative, and in this limit does not depend on the overall electrolyte concentration. The first term in Eq. (C3), proportional to $D_{+}-D_{-}$, is the diffusion potential while the second term is the streaming potential.

\section{Iontophoresis}

In "iontophoresis" a drug is delivered through the skin upon application of an electric field. The effect of advection has previously been studied $[1,64]$ under the "Goldman approximation" of a constant electric field strength [58]. The "iontophoretic enhancement factor" is given by the flux $N_{i}$ relative to the flux $N_{i}(\Delta \phi=\mathrm{Pe}=0)=$ $D_{i} v_{i}\left(c_{l}-c_{r} / L\right)$ in the absence of an electric field and associated flow. This can now be directly calculated from Eq. (A7), without the assumption of a constant electric field, as

$$
\begin{aligned}
\frac{N_{i}}{N_{i}(\Delta \phi=\mathrm{Pe}=0)}= & \frac{\mathrm{Pe}\left(c_{l} e^{\mathrm{Pe}}-c_{r}\right)}{\left(e^{\mathrm{Pe}}-1\right)\left(c_{l}-c_{r}\right)} \\
& \times \frac{\ln \left(c_{r} / c_{l}\right)-D_{a} \mathrm{Pe} / D_{i}+z_{i} F \Delta \phi / R T}{\ln \left(c_{r} / c_{l}\right)-\mathrm{Pe}} .
\end{aligned}
$$

This generalizes the analysis of Kasting and Keister [65] to include advection or the result of Srinivasan and Higuchi [1] to relax the assumption of a constant electric field.

\section{APPENDIX D: SERIES BOUNDARY LAYERS}

In this appendix we consider the typical situation in which a porous layer under consideration is connected through unstirred "Nernst" boundary layers of thickness $\tilde{L}$ with the stirred bulk electrolyte of concentration $c_{0}$, as schematically shown in Fig. 18. For simplicity, here we consider equal bulk concentrations and boundary layer thicknesses on the left and right, which can however be easily generalized. We use a tilde to refer to quantities pertaining to these boundary layers.

In the absence of a net current density in the boundary layers, Eq. (15) gives $\tilde{N}_{i}=v_{i} \tilde{N}$. When the $n$th ion does not react, in steady state $\tilde{N}_{n}=N_{n}$ is a constant throughout the

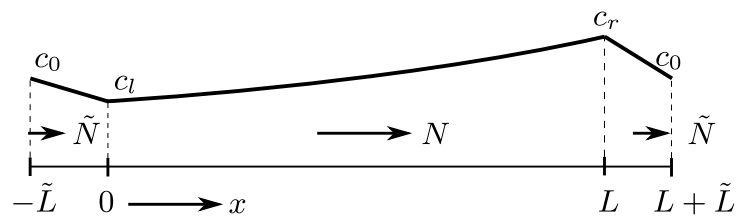

FIG. 18. An extended domain extending the porous layer between $x=0$ and $x=L$ to include two boundary layers of thickness $\tilde{L}$ with electrolyte fluxes $\tilde{N}=D_{a}\left[\left(c_{0}-c_{l}\right) / \tilde{L}\right]=$ $D_{a}\left[\left(c_{r}-c_{0}\right) / \tilde{L}\right]$. 
whole domain. Using this in Eq. (15) gives

$$
\tilde{N}-N=\frac{t_{n} i}{F z_{n} v_{n}} .
$$

In terms of a mass-transfer coefficient $k=D_{a} / \tilde{L}$ we can write $\tilde{N}=k\left(c_{0}-c_{l}\right)=k\left(c_{r}-c_{0}\right)$ so that $c_{l} / c_{r}=$ $\left(1-\tilde{N} / \tilde{N}_{\text {lim }}\right) /\left(1+\tilde{N} / \tilde{N}_{\text {lim }}\right)$ in terms of a maximum flux magnitude $\tilde{N}_{\text {lim }}=k c_{0}$. From Eq. (A2) we have $i_{\text {lim }}=$ $\left(F z_{n} v_{n} / t_{n}\right)\left(\tilde{N}_{\text {lim }}-N_{\text {lim }}\right)$, or, with Eq. (D1),

$$
i_{\lim }=\frac{F z_{n} v_{n} D_{a}}{t_{n}}\left(\frac{c_{0}}{\tilde{L}}+\frac{c_{r}}{L} \frac{\mathrm{Pe}}{e^{\mathrm{Pe}}-1}\right) .
$$

When the flow is to the right, $\mathrm{Pe}>0$, the two contributions of Eq. (D2) add up to increase the limiting current associated with $c_{l}=0$. When $k$ is sufficiently high, $c_{l} \approx c_{r} \approx c_{0}$, allowing very little diffusive transport. The current will in this case be primarily carried by migration and Eq. (24) gives the purely ohmic potential drop $\Delta \phi \approx-i L / \kappa_{0}$.

Note that the boundary layer thickness $\tilde{L}$ used here is a virtual thickness that can be considered to be defined by $\tilde{L}=D_{a} / k$. For a boundary layer with linearly varying concentration of thickness $\delta$ and ambipolar diffusivity $\tilde{D}_{a}$, we have $k=\tilde{D}_{a} / \delta$ so that $\tilde{L}=\delta\left(D_{a} / \tilde{D}_{a} t\right)$. When the effective diffusivity in the porous medium is corrected through Eq. (6) but there is no such correction for the boundary layers, we find that $\tilde{L}=\delta \tau / \epsilon$.

\section{APPENDIX E: TIME-DEPENDENT ANALYTICAL SOLUTIONS}

The general solution to Eq. (29) reads

$$
\frac{c_{l}(t)}{c_{l}(0)}=\left(1+\int_{0}^{t} \frac{\left(1-i / i_{\mathrm{lim}}\right) e^{-\int_{0}^{t^{\prime}} d t^{\prime \prime} / \tau}}{\tau} d t^{\prime}\right) e^{-\int_{0}^{t}\left(d t^{\prime} / \tau\right) d t^{\prime}} .
$$

The steady-state solution of Eq. (29), if it exists, reads $c_{l} / c_{0}=1-i / i_{\mathrm{lim}}$. For ease of notation, we assume that
$V_{l}(0)=V_{r}(0)=V_{0}$. For a constant velocity $u$, Eq. (28) gives $V_{l}(t)=1-t / t_{0}$, where

$$
t_{0}=\frac{V_{0}}{u A}=\frac{2 \tau(0)}{e^{\mathrm{Pe}}-1} .
$$

When $u>0$, this is the time it takes to drain the left volume; when $u<0$, the positive $-t_{0}$ will be the time it takes to drain the right volume. Contrary to Eq. (37) in the main text we thus allow $t_{0}$ to also have a negative sign here. As also remarked in the main text, a steady state is obtained well before full draining occurs only when $\tau(0) \ll t_{0}$, which at equal starting volumes requires $P e$ to be positive but well below 1 . We have $V_{r}=V_{r}(0)\left(1+t / t_{0}\right)$ and $i_{\lim }=i_{\lim }(0) /\left(1+t / t_{0}\right)$ so that

$$
\tau=\tau(0)\left(1-\frac{t^{2}}{t_{0}^{2}}\right)
$$

As one of the volumes empties, therefore, the equilibration time $\tau$ tends to zero. From Eq. (E3), for times $t$ much smaller than the draining time $t_{0}$, we can therefore consider $\tau$ and $i_{\text {lim }}$ to be constant so that Eq. (E1) reduces to the simple exponential decay equation; for $t \ll t_{0}$,

$$
\frac{c_{l}(t)}{c_{l}(0)} \approx 1-\frac{i}{i_{\lim }(0)}\left(1-e^{-t / \tau(0)}\right)
$$

Since according to Eq. (E3) $\tau$ varies quadratically with $t / t_{0}$ while $1 / i_{\text {lim }}$ varies linearly with $t / t_{0}$, for short times, we can take $\tau$ to be constant so that, for $t^{2} \ll t_{0}^{2}$,

$$
\frac{c_{l}(t)}{c_{l}(0)} \approx 1-\frac{i}{i_{\lim }(0)}\left(1+\frac{t-\tau-\left(t_{0}-\tau\right) e^{-t / \tau}}{t_{0}}\right)
$$

Inserting a posteriori the time dependence of $\tau$ in Eq. (E5) can arguably improve the solution somewhat as $t$ approaches $t_{0}$, as shown in Fig. 19.

Taking $i_{\text {lim }}$ constant in Eq. (E1) but taking into account the time dependence of $\tau$, replace $e^{-t / \tau}$ with $e^{-\int_{0}^{t} d t^{\prime} / \tau\left(t^{\prime}\right)}=$ $e^{-\operatorname{atanh} t / \tau(0)}=\left[\left(1-t / t_{0}\right) /\left(1+t / t_{0}\right)\right]^{t_{0} / 2 \tau(0)}$. Making this replacement in Eq. (E5) gives

$$
\frac{c_{l}(t)}{c_{l}(0)} \approx 1-\frac{i}{i_{\lim }(0)}\left(1+\frac{t-\tau-\left(t_{0}-\tau\right)\left[\left(1-t / t_{0}\right) /\left(1+t / t_{0}\right)\right]^{t_{0} / 2 \tau(0)}}{t_{0}}\right) .
$$

This can slightly further improve upon Eq. (E5), as shown in Fig. 19.

An exact solution is possible for integer values of $t_{0} / \tau(0)$. For example, when $\mathrm{Pe}=\ln 3 \approx 1.1$, the exact solution for $t_{0}=\tau(0)$ can be shown to be

$$
\frac{c(t)}{c(0)}=1+\frac{2 i}{i_{\lim }(0)}\left\{\left[1+\frac{1}{2} \arcsin \left(\frac{t}{t_{0}}\right)\right] \sqrt{\frac{t_{0}-t}{t_{0}+t}}-1\right\} .
$$




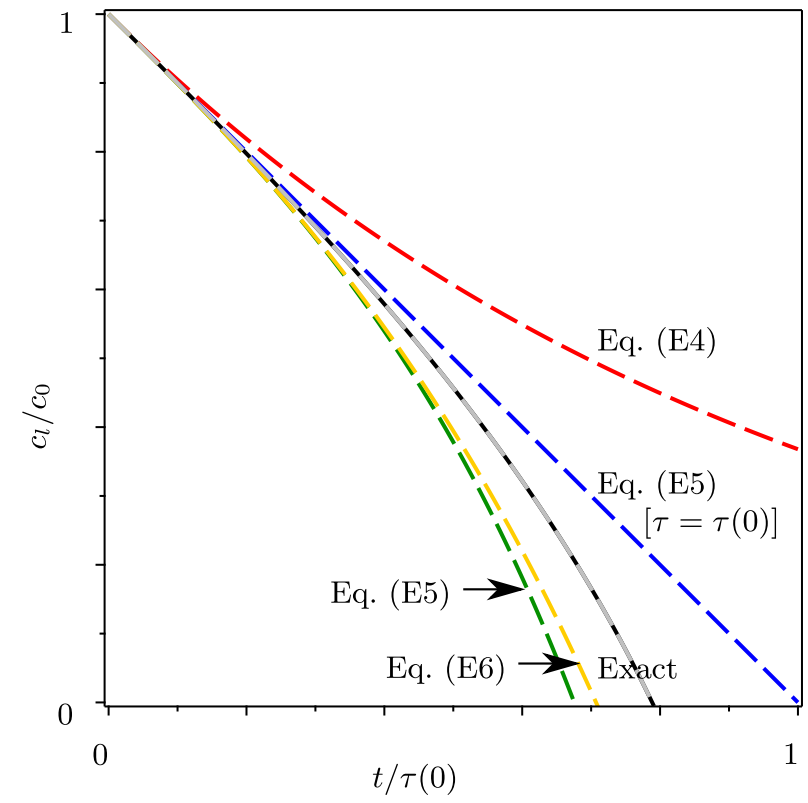

FIG. 19. A comparison between the analytical (dashed) and numerical (solid) solution of Eq. (29) for the left electrolyte concentration $c_{l}$ normalized by the initial concentration $c_{l}(0)=$ $c_{r}(0)=c_{0}$ for $V_{l}(0)=V_{r}(0)=V_{0}, i=i_{\text {lim }}(0)$, and a constant $\mathrm{Pe}=\ln 3 \approx 1.1$, so that, from Eq. (E2), the time to draining $t_{0}=\tau(0)$. Note that the concentration on the left depletes before full draining occurs, because the limiting current $i_{\text {lim }}$ decreases as $V_{r}$ increases. The exact analytical result of Eq. (E7) overlaps with the numerical result.

This solution exactly overlaps with the numerical solution in Fig. 19. When $\mathrm{Pe}=\ln 2 \approx 0.7$, an exact solution for $t_{0}=2 \tau(0)$ reads

$$
\frac{c(t)}{c(0)}=1+\frac{4 i}{i_{\lim }(0)} \frac{\left(1-t / t_{0}\right)\left[1-\ln \left(1-t / t_{0}\right) / 2\right]-1}{1+t / t_{0}} .
$$

This solution is plotted in Fig. 5 along with various quantities derived from it and is compared to the numerical solution in which $\mathrm{Pe}$ is not constant but evolves according to Eq. (32).

In case of zero flow, with $V_{l}=V_{r}=V_{0}$ and $c_{l}(0)=$ $c_{r}(0)$, we can insert Eq. (E4) with constant $\tau=\tau(0)$ into Eq. (26) to give

$$
\Delta \phi=\left(\chi+\frac{i_{\lim } L}{2 \kappa_{0}} \frac{1}{1-e^{-t / \tau}}\right) \ln \left(\frac{i_{\lim }-i\left(1-e^{-t / \tau}\right)}{i_{\lim }+i\left(1-e^{-t / \tau}\right)}\right)
$$

where we have used Eqs. (21) and (E4) and $c_{0}=$ $\left(c_{l}+c_{r}\right) / 2$ to write $N / N_{L}=\left(i / i_{\lim }\right)\left(1-e^{-t / \tau}\right)$. For small times, expanding around $t=0$ gives

$$
-\Delta \phi \approx \frac{i L}{\kappa_{0}}+2 \chi \frac{i}{i_{\lim }} \frac{t}{\tau}=\frac{i L}{\kappa_{0}}+\frac{2 t_{n} \chi}{z_{n} v_{n} F c_{0} V_{0}} i A t \quad(t \ll \tau),
$$

where the first term is the ohmic potential drop and the second term is that of a charging capacitor with capacitance $v_{n} z_{n} F c_{0} V_{0} / 2 \chi t_{n}$. This capacitance is equal to the average electrolyte charge on either side, divided by the constant potential $2 t_{n} \chi$.

\section{APPENDIX F: CONCENTRATION OVERPOTENTIAL}

The current density $i$ when driven by an electrochemical reaction, can be increased by increasing the applied cell potential $E_{\text {cell }}$ with an "overpotential" $\eta$ beyond the opencircuit potential $E_{\mathrm{oc}}$ required for $i=0$. This dependence can often be described by the concentration-dependent Butler-Volmer equation [32]

$$
\begin{aligned}
i & =i_{* l}\left(\frac{c_{l}}{c_{0}} e^{\eta_{l} / b_{l}}-e^{-\left[\left(1-\alpha_{l}\right) / \alpha_{l}\right]\left(\eta_{l} / b_{l}\right)}\right) \\
& =i_{* r}\left(e^{\eta_{r} / b_{r}}-\frac{c_{r}}{c_{0}} e^{-\left[\left(1-\alpha_{r}\right) / \alpha_{r}\right]\left(\eta_{r} / b_{r}\right)}\right) .
\end{aligned}
$$

The exchange current densities $i_{* l}$ and $i_{* r}$ of the left and right electrodes are a combined measure of the intrinsic reaction rate and the total reactive surface area available. The Tafel slopes $b_{l}=R T / \alpha_{l} F$ and $b_{r}=R T / \alpha_{r} F$ are related to the charge transfer coefficients $\alpha_{l}$ and $\alpha_{r}$, which typically do not deviate strongly from $\frac{1}{2}$. In Eq. (F1), we assumed that the left forward and right backward reactions are first order in the electrolyte concentration. This is, for example, the case when on the left electrode the oxygen evolution reaction $2 \mathrm{OH}^{-} \rightarrow 2 e^{-}+\frac{1}{2} \mathrm{O}_{2}+\mathrm{H}_{2} \mathrm{O}$ takes place while on the right electrode the hydrogen evolution reaction $2 \mathrm{H}_{2} \mathrm{O}+2 e^{-} \rightarrow 2 \mathrm{OH}^{-}+\mathrm{H}_{2}$ occurs, and $c=$ $\left[\mathrm{OH}^{-}\right]$. The dissolved reaction products are here assumed to be present at a constant concentration. In the Tafel regime $F \eta_{l} / R T \gtrsim \ln \left(c_{0} / c_{l}\right)$ and $F \eta_{r} / R T \gtrsim \ln \left(c_{r} / c_{0}\right)$ we can neglect the backward reaction and write

$$
i=\mathcal{E}_{l} i_{* l} \frac{c_{l}}{c_{0}} e^{\eta_{l} / b_{l}}=\mathcal{E}_{r} i_{* r} e^{\eta_{r} / b_{r}}
$$

where we account for effectiveness factors $\mathcal{E}_{l}, \mathcal{E}_{r} \leq 1$ reducing the current, relative to the maximum current in the case of a homogeneous reaction [38]. If we assign to the left and right electrodes a potential of $E_{\text {cell }}$ and 0 , respectively, the left and right overpotentials are given by the difference between the electrode and electrolyte potentials as $\eta_{l}=E-E_{\mathrm{oc}}-\phi(0)$ and $\eta_{r}=\phi(L)$, respectively, with $E_{\text {cell }}$ the total cell potential and $E_{\mathrm{oc}}$ the open-circuit potential at which $i=0$, which we assume here to be equal to 
the equilibrium potential of the reaction. Therefore, with $\Delta \phi=\phi(L)-\phi(0)$ we can write

$$
E_{\mathrm{cell}}=E_{\mathrm{oc}}-\Delta \phi+b \ln \left(\frac{i}{i_{*}}\right)+b_{l} \ln \left(\frac{c_{0}}{c_{l}}\right),
$$

where $\quad b=b_{l}+b_{r}=\left[\left(\alpha_{l}+\alpha_{r}\right) / \alpha_{l} \alpha_{r}\right](R T / F), \quad i_{*}=$ $\left(i_{* l}^{\alpha_{r}} i_{* r}^{\alpha_{l}}\right)^{1 /\left(\alpha_{l}+\alpha_{r}\right)}, \quad \mathcal{E}=\left(\mathcal{E}_{l}^{\alpha_{r}} \mathcal{E}_{r}^{\alpha_{l}}\right)^{1 /\left(\alpha_{l}+\alpha_{r}\right)}$ are the effective combined Tafel slope, exchange current density, and effectiveness factors, respectively.

When for small overpotentials $\eta_{l}, \eta_{r} \lesssim R T / F$ it holds that $c_{l} \approx c_{r} \approx c_{0}$, Eq. (F1) can be approximated by $i=$ $2 i_{* l}\left(c_{l} / c_{0}\right) \sinh \left(\eta_{l} / b_{l}\right)=2 i_{* r} \sinh \left(\eta_{r} / b_{r}\right)$ when $\alpha_{l}=\alpha_{r}=$ $\frac{1}{2}$. Under these special circumstances, Eq. (F1) gives

$E_{\mathrm{cell}} \approx E_{\mathrm{oc}}-\Delta \phi+b_{l} \operatorname{asinh}\left(\frac{i}{2 i_{* l}} \frac{c_{0}}{c_{l}}\right)+b_{r} \operatorname{asinh}\left(\frac{i}{2 i_{* r}}\right)$.

Equation (F1) or either of its explicit approximations, Eq. (F3) or (F4), can be used in combination with Eq. (24) for $\Delta \phi$ and differential equations (28) and (29) for $c_{l}$ to give a complete set of equations describing the dynamic evolution of the cell potential as a function of time.

The final term of Eq. (F3) is the concentration overpotential. Inserting Eq. (E4) gives, for $t \ll t_{0}$,

$$
\Delta E_{\mathrm{conc}}=b_{a} \ln \left(\frac{c_{0}}{c_{a}}\right) \approx-b_{a} \ln \left(1-\frac{i\left(1-e^{-t / \tau(0)}\right)}{i_{\mathrm{lim}}(0)}\right) .
$$

When $i=i_{\text {lim }}(0)$, the concentration overpotential $\Delta E_{\text {conc }} /$ $b_{a}=t / \tau(0)$ increases linearly with time, at a rate of one Tafel slope per time $\tau(0)$. Note that, since $p \mathrm{H}$ also logarithmically relates to $c_{l}$, this will vary linearly with time initially in this case; see Fig. 14.

[1] V. Srinivasan and W. I. Higuchi,A model for iontophoresis incorporating the effect of convective solvent flow,Int. J. Pharm. 60,133(1990).

[2] R. Telaranta, J. A. Manzanares, and K. Kontturi, Convective electrodiffusion processes through graft-modified charged porous membranes, J. Electroanal. Chem. 464, 222 (1999).

[3] Anna-Kaisa Kontturi, Kyösti Kontturi, José A. Manzanares, and Salvador Mafé, Equilibrium and transport properties of polydisperse polyelectrolytes in graftmodified porous charged membranes: Forced permeation-diffusion of lignosulfonate, Phys. Chem. Chem. Phys. 1, 1939 (1999).

[4] Mohammad R. Hossan, Diganta Dutta, Nazmul Islam, and Prashanta Dutta, Electric field driven pumping in microfluidic device, Electrophoresis 39, 702 (2018).

[5] E. K. Zholkovskii, Solvent transport through membranes during electrodialysis, Sov. Electrochem. 28, 158 (1992).
[6] Yoshinobu Tanaka, Concentration polarization in ion exchange membrane electrodialysis, J. Membrane Sc. 57, 217 (1991).

[7] M. W. Vonk and J. A. M. Smit, The application of generalized Nernst-Planck equations to the description of ion retention in the hyperfiltration of mixed electrolyte solutions through a neutral membrane, Berich. Bunsen Gesell. 88, 724 (1984).

[8] Andriy E. Yaroshchuk, Negative rejection of ions in pressure-driven membrane processes, Adv. Colloid Interf. Sc. 139, 150 (2008).

[9] Tatsuya Shinagawa and Kazuhiro Takanabe, Towards versatile and sustainable hydrogen production through electrocatalytic water splitting: Electrolyte engineering, ChemSusChem 10, 1318 (2017).

[10] Pablo Peñas, Peter van der Linde, Wouter Vijselaar, Devaraj van der Meer, Detlef Lohse, Jurriaan Huskens, Han Gardeniers, Miguel A. Modestino, and David Fernández Rivas, Decoupling gas evolution from water-splitting electrodes, J. Electrochem. Soc. 166, H769 (2019).

[11] Vincent Fleury, James Kaufman, and Brynn Hibbert, Evolution of the space-charge layer during electrochemical deposition with convection, Phys. Rev. E 48, 3831 (1993).

[12] Christophe Léger, J. Elezgaray, and F. Argoul, Dynamical characterization of one-dimensional stationary growth regimes in diffusion-limited electrodeposition processes, Phys. Rev. E 58, 7700 (1998).

[13] William A. Braff, Cullen R. Buie, and Martin Z. Bazant, Boundary layer analysis of membraneless electrochemical cells, J. Electrochem. Soc. 160, A2056 (2013).

[14] Christoffer P. Nielsen and Henrik Bruus, Concentration polarization, surface currents, and bulk advection in a microchannel, Phys. Rev. E 90, 043020 (2014).

[15] Aditya S. Khair, Concentration polarization and secondkind electrokinetic instability at an ion-selective surface admitting normal flow, Phys. Fluids 23, 072003 (2011).

[16] Andriy Yaroshchuk, What makes a nano-channel? A limiting-current criterion, Microfl. Nanofl. 12, 615 (2012).

[17] Remco Hartkamp, Bertrand Siboulet, Jean-François Dufrêche, and Benoit Coasne, Ion-specific adsorption and electroosmosis in charged amorphous porous silica, Phys. Chem. Chem. Phys. 17, 24683 (2015).

[18] Inhee Cho, Wonseok Kim, Junsuk Kim, Ho-Young Kim, Hyomin Lee, and Sung Jae Kim, Non-Negligible DiffusioOsmosis inside an ion Concentration Polarization Layer, Phys. Rev. Lett. 116, 254501 (2016).

[19] E Victoria Dydek, Boris Zaltzman, Isaak Rubinstein, D. S. Deng, Ali Mani, and Martin Z. Bazant, Overlimiting Current in a Microchannel, Phys. Rev. Lett. 107, 118301 (2011).

[20] Isaak Rubinstein and Frank Maletzki, Electroconvection at an electrically inhomogeneous permselective membrane surface, J. Chem. Soc. Faraday Trans. 87, 2079 (1991).

[21] Shmuel M. Rubinstein, Gor Manukyan, Adrian Staicu, Issac Rubinstein, Boris Zaltzman, Rob G. H. Lammertink, Frieder Mugele, and Matthias Wessling, Direct Observation of a Nonequilibrium Electro-Osmotic Instability, Phys. Rev. Lett. 101, 236101 (2008). 
[22] I. Rubinstein and B. Zaltzman, Equilibrium Electroconvective Instability, Phys. Rev. Lett. 114, 114502 (2015).

[23] Elif Karatay, Mathias Bækbo Andersen, Matthias Wessling, and Ali Mani, Coupling between Buoyancy Forces and Electroconvective Instability Near Ion-Selective Surfaces, Phys. Rev. Lett. 116, 194501 (2016).

[24] Andriy Yaroshchuk, Over-limiting currents and deionization "shocks" in current-induced polarization: Localequilibrium analysis, Adv. Colloid Interface Sci. 183, 68 (2012).

[25] E. Victoria Dydek and Martin Z. Bazant, Nonlinear dynamics of ion concentration polarization in porous media: The leaky membrane model, AIChE J. 59, 3539 (2013).

[26] Sung Jae Kim, Ying-Chih Wang, Jeong Hoon Lee, Hongchul Jang, and Jongyoon Han, Concentration Polarization and Nonlinear Electrokinetic Flow Near a Nanofluidic Channel, Phys. Rev. Lett. 99, 044501 (2007).

[27] Leon Rosentsvit, Sinwook Park, and Gilad Yossifon, Effect of advection on transient ion concentration-polarization phenomenon, Phys. Rev. E 96, 023104 (2017).

[28] Ali Mani and Martin Z. Bazant, Deionization shocks in microstructures, Phys. Rev. E 84, 061504 (2011).

[29] Jesús Rodríguez, Simonetta Palmas, Margarita SánchezMolina, Ernesto Amores, Laura Mais, and Roberto Campana, Simple and precise approach for determination of ohmic contribution of diaphragms in alkaline water electrolysis, Membranes 9, 129 (2019).

[30] M. G. H. M. Hendriks, Johan E. ten Elshof, Henricus J. M. Bouwmeester, and H. Verweij, The electrochemical doublelayer capacitance of yttria-stabilised zirconia, Solid State Ionics 146, 211 (2002).

[31] J. W. Haverkort and H. Rajaei, Electroosmotic flow and the limiting current in alkaline water electrolysis, J. Power Sources Adv. 6, 100034 (2020).

[32] John Newman and Karen E. Thomas-Alyea, Electrochemical Systems (John Wiley \& Sons, New Jersey, 2012).

[33] Sungmin Nam, Inhee Cho, Joonseong Heo, Geunbae Lim, Martin Z. Bazant, Dustin Jaesuk Moon, Gun Yong Sung, and Sung Jae Kim, Experimental Verification of Overlimiting Current by Surface Conduction and Electro-Osmotic Flow in Microchannels, Phys. Rev. Lett. 114, 114501 (2015).

[34] Evgeniy Boyko, Ran Eshel, Amir D. Gat, and Moran Bercovici, Nonuniform Electro-Osmotic Flow Drives Fluid-Structure Instability, Phys. Rev. Lett. 124, 024501 (2020).

[35] Vicente M. Aguilella, Salvador Mafé, and Julio Pellicer, Ionic transport through a homogeneous membrane in the presence of simultaneous diffusion, conduction and convection, J. Chem. Soc. Faraday Trans. 1 85, 223 (1989).

[36] M. Paulin, D. Hutin, and F. Coeuret, Theoretical and experimental study of flow-through porous electrodes, J. Electrochem. Soc. 124, 180 (1977).

[37] K. Scott, Short communication the effectiveness of particulate bed electrodes under activation control, Electrochim. Acta 27, 447 (1982).

[38] J. W. Haverkort, A theoretical analysis of the optimal electrode thickness and porosity, Electrochim. Acta 295, 846 (2019).
[39] R. N. Bhatia, K. E. Gubbins, and R. D. Walker, Mutual diffusion in concentrated aqueous potassium hydroxide solutions, Trans. Faraday Soc. 64, 2091 (1968).

[40] R. J. Gilliam, J. W. Graydon, D. W. Kirk, and S. J. Thorpe, A review of specific conductivities of potassium hydroxide solutions for various concentrations and temperatures, Int. J. Hydrog. Energy 32, 359 (2007).

[41] John S. Newman and Charles W. Tobias, Theoretical analysis of current distribution in porous electrodes, J. Electrochem. Soc. 109, 1183 (1962).

[42] S. A. Grigoriev, P. Millet, S. V. Korobtsev, V. I. Porembskiy, M. Pepic, C. Etievant, C. Puyenchet, and V. N. Fateev, Hydrogen safety aspects related to high-pressure polymer electrolyte membrane water electrolysis, Int. J. Hydrog. Energy 34, 5986 (2009).

[43] Richard Buchner, Glenn Hefter, Peter M. May, and Pál Sipos, Dielectric relaxation of dilute aqueous $\mathrm{NaOH}$, $\mathrm{NaAl}(\mathrm{OH})_{4}$, and $\mathrm{NaB}(\mathrm{OH})_{4}$, J. Phys. Chem. B 103, 11186 (1999).

[44] Alan K. Soper and Kristian Weckström, Ion solvation and water structure in potassium halide aqueous solutions, Biophys. Chem. 124, 180 (2006).

[45] Frank H. J. Van der Heyden, Derek Stein, Koen Besteman, Serge G. Lemay, and Cees Dekker, Charge Inversion at High Ionic Strength Studied by Streaming Currents, Phys. Rev. Lett. 96, 224502 (2006).

[46] Maximilian Schalenbach, Wiebke Lueke, and Detlef Stolten, Hydrogen diffusivity and electrolyte permeability of the Zirfon PERL separator for alkaline water electrolysis, J. Electrochem. Soc. 163, F1480 (2016).

[47] Geoffrey Ingram Taylor, Dispersion of soluble matter in solvent flowing slowly through a tube, Proc. R. Soc. London A 219, 186 (1953).

[48] Andriy Yaroshchuk, Emiliy Zholkovskiy, Sergey Pogodin, and Vladimir Baulin, Coupled concentration polarization and electroosmotic circulation near micro/nanointerfaces: Taylor-Aris model of hydrodynamic dispersion and limits of its applicability, Langmuir 27, 11710 (2011).

[49] P. Trinke, P. Haug, J. Brauns, B. Bensmann, R. HankeRauschenbach, and T. Turek, Hydrogen crossover in PEM and alkaline water electrolysis: Mechanisms, direct comparison and mitigation strategies, J. Electrochem. Soc. 165, F502 (2018).

[50] Philipp Haug, Matthias Koj, and Thomas Turek, Influence of process conditions on gas purity in alkaline water electrolysis, Int. J. Hydrog. Energy 42, 9406 (2017).

[51] A. M. Peers, Membrane phenomena, Discuss. Faraday Soc. 21, 124 (1956).

[52] Ernst Baars, in Handbuch der Physik (Springer, 1928), pp. 517-627.

[53] Artjom V. Sokirko and Fritz H. Bark, Diffusion-migration transport in a system with Butler-Volmer kinetics, an exact solution, Electrochim. Acta 40, 1983 (1995).

[54] Veniamin Grigor'evich Levich, Physicochemical Hydrodynamics (Prentice-Hall Inc., New Jersey, 1962).

[55] P. M. Biesheuvel, M. Van Soestbergen, and M. Z. Bazant, Imposed currents in galvanic cells, Electrochim. Acta 54, 4857 (2009).

[56] V. V. Nikonenko, K. A. Lebedev, and S. S. Suleimanov, Influence of the convective term in the nernst-planck 
equation on properties of ion transport through a layer of solution or membrane, Russ. J. Electrochem. 45, 160 (2009).

[57] Xavier Lefebvre, John Palmeri, and Patrice David, Nanofiltration theory: An analytic approach for single salts, J. Phys. Chem. B 108, 16811 (2004).

[58] David E. Goldman, Potential, impedance, and rectification in membranes, J. Gen. Physiol. 27, 37 (1943).

[59] R. Schlögl, Membrane permeation in systems far from equilibrium, Berich Bunsen Gesell. 70, 400 (1966).

[60] P. M. Biesheuvel, Two-fluid model for the simultaneous flow of colloids and fluids in porous media, J. Colloid Interface Sci. 355, 389 (2011).

[61] Andriy Yaroshchuk and Mykola P. Bondarenko, Currentinduced concentration polarization of nanoporous media: Role of electroosmosis, Small 14, 1703723 (2018).
[62] K. S. Spiegler and O. Kedem, Thermodynamics of hyperfiltration (reverse osmosis): Criteria for efficient membranes, Desalination 1, 311 (1966).

[63] Carsten Werner, Heinz Körber, Ralf Zimmermann, Stanislav Dukhin, and Hans-Jörg Jacobasch, Extended electrokinetic characterization of flat solid surfaces, J. Colloid Interface Sci. 208, 329 (1998).

[64] Bradley D. Bath, Henry S. White, and Erik R. Scott, Electrically facilitated molecular transport. Analysis of the relative contributions of diffusion, migration, and electroosmosis to solute transport in an ion-exchange membrane, Anal. Chem. 72, 433 (2000).

[65] Gerald B. Kasting and J. C. Keister, Application of electrodiffusion theory for a homogeneous membrane to iontophoretic transport through skin, J. Control. Release 8, 195 (1989). 